\title{
The Local Average Contact (LAC) method
}

\author{
M. Abbas ${ }^{\mathrm{a}, \mathrm{b}}$, G. Drouet ${ }^{\mathrm{a}, \mathrm{b}, \mathrm{c}}$, P. Hild ${ }^{\mathrm{c}}$ \\ ${ }^{a}$ IMSIA, UMR 9219 EDF-CNRS-CEA-ENSTA, Université Paris Saclay, 828 Boulevard \\ des Maréchaux, 91762 Palaiseau Cedex France \\ ${ }^{b}$ Électricité de France Recherche et Développement, 7 Boulevard Gaspard Monge, 91120 \\ Palaiseau, France \\ ${ }^{c}$ Institut de Mathématiques de Toulouse ; UMR 5219, Université de Toulouse ; CNRS ; \\ UPS-IMT, 118 route de Narbonne, 31062 Toulouse Cedex 9, France
}

\begin{abstract}
The Local Average Contact (LAC) method allows the handling of nonmatching meshes in an easy way by averaging locally the interpenetration between the contacting bodies. In this paper we consider several numerical experiments involving two and three-dimensional bodies discretized with various linear and quadratic finite elements. We also present a convergence analysis of the method in the geometrical nonconforming case in which the boundary points of the candidate contact areas do not coincide.
\end{abstract}

Keywords: Local Average Contact (LAC) condition, Unilateral contact, nonmatching meshes, geometrical nonconformity.

\section{Introduction}

Finite element methods are currently used to approximate the unilateral contact problems in solid mechanics (see, e.g., $[1,2,3,4,5]$ ). Such problems show a nonlinear boundary condition, which requires that the normal component of the relative displacement field of the contacting bodies is nonpositive on a part of their boundaries (see [6]). This nonlinearity leads to a weak formulation written as a variational inequality which admits a unique solution (see [7]) and the regularity of the solution shows limitations whatever the regularity of the data is (see [8]). A consequence is that only finite element

Email addresses: mickael.abbas@edf.fr (M. Abbas), guillaume.drouet@edf.fr (G. Drouet), patrick.hild@math.univ-toulouse.fr (P. Hild) 
methods of order one and of order two are of interest which is the scope of this work.

The present paper focuses on the contact problems between two or more bodies whose respective meshes may not coincide on the contact interface, the so called "nonmatching meshes" or "noncoinciding meshes". This situation which often occurs in engineering computations (since the bodies are usually meshed independently) has been considered from a numerical and theoretical point of view in the last twenty years. It is now known that the local node-on-segment contact conditions in 2D or the equivalent nodeon-face conditions in 3D produce solutions with oscillations which degrade the accuracy and slow down the convergence of the computations. On the contrary the mortar domain decomposition method [9] handles in an optimal way the nonmatching meshes and its adaptation to contact problems gave promising theoretical and numerical results at the end of the 90's (see $[10,11,12,13,14])$. To summarize, this initial approach directly inspired from [9], considered a global $L^{2}$-projection of linear finite element functions from a mesh to another mesh on the contact area in two-dimensions. From a numerical point of view, this mortar concept has been adapted and extended to many contact configurations such as friction, quadratic finite elements, large deformations, three-dimensional problems... see, e.g. $[15,16,17,18,19,20,21,22,23,24,25,26,27,28]$ and the references therein.

The Local Average Contact (LAC) method introduced in [29] is an approach handling in a local way the contact constraint by averaging locally the jump of the normal displacement denoted $\left[u_{N}^{h}\right]$ independently of the space dimension and of the degree and type of the finite elements. In a similar way, the LAC method can be seen as a Lagrange method in which the multiplier representing the contact pressure is piecewise constant independently of the degree (one or two) of the finite elements chosen for the displacements. This method provides optimal convergence results in the energy norm in the general case of nonmatching meshes (see the analysis in [29] where the problem is written as a variational inequality) and therefore combines both the advantages of locality and accuracy. In this paper we now consider the LAC approximation using mixed finite elements with Lagrange multipliers, we recover the optimal results of [29] and we show on various examples the capabilities of the method. In addition we focus on the theoretical and practical handling of geometrical nonconformity in which the boundary of the candidate contact area (which is of dimension $d-2$ if the problem is $d$-dimensional) 
does not coincide with any of the discretizations.

The paper is organized as follows: section 2 deals with the equations and the weak formulations modeling the unilateral contact problem between two elastic bodies in linear elasticity. In section 3, the LAC method is introduced. A special attention is paid to the geometrical nonconforming case for which a first convergence analysis is carried out. Section 4 is concerned in particular with the generalized Newton algorithm used to solve the problem and in section 5, we show various numerical results in order to check the capabilities of the method: a standard Taylor patch test in $2 \mathrm{D}$ and $3 \mathrm{D}$ and similar tests with curved boundaries with self included disks and self included spheres. Then we compute numerical convergence rates in 2D and 3D using examples where the exact solutions are known. Finally we consider a more sophisticated three dimensional example, the contact of a deformable ring on a deformable block which is computed using different finite elements $(8,20$ and 27 node hexahedra).

\section{Problem set-up}

\subsection{Spaces and norms}

First, we specify some notations we shall use. Let $\omega$ be a Lebesguemeasurable subset of $\mathbb{R}^{d}, d \geq 1$ with nonempty interior ; the generic point

of $\omega$ is denoted $x$. The classical Lebesgue spaces $L^{p}(\omega), 1<p<\infty$ and the standard Sobolev space $H^{m}(\omega), m \in \mathbb{N}$ (we adopt the convention $H^{0}(\omega)=$ $\left.L^{2}(\omega)\right)$ are endowed with the norms:

$$
\|\psi\|_{L^{p}(\omega)}=\left(\int_{\omega}|\psi(x)|^{p} d x\right)^{1 / p}, \quad\|\psi\|_{m, \omega}=\left(\sum_{0 \leq|\alpha| \leq m}\left\|\partial^{\alpha} \psi\right\|_{L^{2}(\omega)}^{2}\right)^{1 / 2}
$$

where $\alpha=\left(\alpha_{1}, \ldots, \alpha_{d}\right)$ is a multi-index in $\mathbb{N}^{d},|\alpha|=\alpha_{1}+\cdots+\alpha_{d}$ and the symbol $\partial^{\alpha}$ represents a partial derivative. The fractional Sobolev space $H^{\tau}(\omega), \tau \in \mathbb{R}_{+} \backslash \mathbb{N}$ with $\tau=m+\nu, m$ being the integer part of $\tau$ and $\nu \in(0,1)$ is defined by the norm $\|\cdot\|_{\tau, \omega}$ involving the semi-norm $|\cdot|_{\nu, \omega}$ :

$\|\psi\|_{\tau, \omega}=\left(\|\psi\|_{m, \omega}^{2}+\sum_{|\alpha|=m}\left|\partial^{\alpha} \psi\right|_{\nu, \omega}^{2}\right)^{1 / 2},|\psi|_{\nu, \omega}=\left(\int_{\omega} \int_{\omega} \frac{(\psi(x)-\psi(y))^{2}}{|x-y|^{d+2 \nu}} d x d y\right)^{1 / 2}$ 


\subsection{Formulation of contact problem}

Let $\Omega^{1}$ and $\Omega^{2}$ in $\mathbb{R}^{d}, d=2,3$ stand for two domains representing the reference configurations of two linearly elastic bodies. The boundaries $\partial \Omega^{\ell}, \ell=1,2$ consist of three nonoverlapping open parts $\Gamma_{N}^{\ell}, \Gamma_{D}^{\ell}$ and $\Gamma_{C}^{\ell}$ where $\overline{\Gamma_{N}^{\ell}} \cup \overline{\Gamma_{D}^{\ell}} \cup \overline{\Gamma_{C}^{\ell}}=\partial \Omega^{\ell}$. We assume that the measures in $\mathbb{R}^{d-1}$ of $\Gamma_{C}^{\ell}$ and $\Gamma_{D}^{\ell}$ are positive and we set

$$
\Gamma_{C}=\Gamma_{C}^{1} \cap \Gamma_{C}^{2},
$$

$\Gamma_{C}$ is the initial common contact area. The bodies are submitted to a Neumann condition on $\Gamma_{N}^{\ell}$ with a density of loads $F_{\ell} \in\left(L^{2}\left(\Gamma_{N}^{\ell}\right)\right)^{d}$, a Dirichlet condition on $\Gamma_{D}^{\ell}$ (the bodies are assumed to be clamped on $\Gamma_{D}^{\ell}$ to simplify) and to volume loads denoted $f_{\ell} \in\left(L^{2}\left(\Omega^{\ell}\right)\right)^{d}$ in $\Omega^{\ell}$. Finally, a (frictionless) unilateral contact condition between the bodies holds on $\Gamma_{C}$.

The problem consists in finding the displacement field $u=\left(u_{1}, u_{2}\right)$ : $\overline{\Omega^{1}} \times \overline{\Omega^{2}} \rightarrow \mathbb{R}^{d}$ satisfying (1)-(7) with $\ell=1,2$ :

$$
\begin{aligned}
-\operatorname{div} \sigma_{\ell}\left(u_{\ell}\right)=f_{\ell} & \text { in } \Omega^{\ell}, \\
\sigma\left(u_{\ell}\right)=\mathcal{A}_{\ell} \varepsilon\left(u_{\ell}\right) & \text { in } \Omega^{\ell}, \\
\sigma_{\ell}\left(u_{\ell}\right) n_{\ell}=F_{\ell} & \text { on } \Gamma_{N}^{\ell}, \\
u_{\ell}=0 & \text { on } \Gamma_{D}^{\ell},
\end{aligned}
$$

where $n_{\ell}$ stands for the outward unit normal to $\Omega^{\ell}$ on $\partial \Omega^{\ell}$. On $\Gamma_{C}^{\ell} \backslash \Gamma_{C}$ we simply set homogeneous Neumann conditions :

$$
\sigma_{\ell}\left(u_{\ell}\right) n_{\ell}=0 \quad \text { on } \Gamma_{C}^{\ell} \backslash \Gamma_{C}
$$

On $\Gamma_{C}$, we decompose the displacement and the stress vector fields in normal and tangential components as follows:

$$
\begin{gathered}
u_{\ell_{N}}=u_{\ell} \cdot n_{\ell}, \quad u_{\ell_{T}}=u_{\ell}-u_{\ell_{N}} n_{\ell}, \\
\sigma_{\ell_{N}}=\left(\sigma_{\ell}\left(u_{\ell}\right) n_{\ell}\right) \cdot n_{\ell}, \quad \sigma_{\ell_{T}}=\sigma_{\ell}\left(u_{\ell}\right) n_{\ell}-\sigma_{\ell_{N}} n_{\ell},
\end{gathered}
$$

and we denote by

$$
\left[u_{N}\right]=u_{1_{N}}+u_{2_{N}}
$$

the jump of the normal displacement across the contact interface. 
The unilateral contact condition on $\Gamma_{C}$ is expressed by the following complementarity condition:

$$
\left[u_{N}\right] \leq 0, \quad \sigma_{1_{N}}=\sigma_{2_{N}}=\sigma_{N} \leq 0, \quad\left[u_{N}\right] \sigma_{N}=0
$$

where a vanishing gap between the two elastic solids has been chosen in the reference configuration.

The frictionless condition on $\Gamma_{C}$ reads as: for $\ell=1,2$

$$
\sigma_{\ell_{T}}=0 \text {. }
$$

We introduce the spaces $V_{\ell}=\left\{v_{\ell} \in\left(H^{1}\left(\Omega^{\ell}\right)\right)^{d}: v=0\right.$ on $\left.\Gamma_{D}^{\ell}\right\}$ and the product space

$$
V=V_{1} \times V_{2}
$$

endowed with the broken norm

$$
\|v\|=\left(\sum_{\ell=1}^{2}\left\|v_{\ell}\right\|_{1, \Omega^{\ell}}^{2}\right)^{\frac{1}{2}}, \quad \forall v=\left(v_{1}, v_{2}\right) \in V .
$$

The forthcoming mixed variational formulation uses the convex cone $M$ of multipliers on $\Gamma_{C}$

$$
M=\left\{\mu \in W^{\prime},\langle\mu, \psi\rangle_{W^{\prime}, W} \geq 0 \text { for all } \psi \in W, \psi \leq 0 \text { a.e. on } \Gamma_{C}\right\},
$$

where $W \subset H^{\frac{1}{2}}\left(\Gamma_{C}\right)$ is the range of $V$ by the normal trace operator on $\Gamma_{C}$, $W^{\prime}$ denotes its dual and $\langle.,\rangle_{W^{\prime}, W}$ stands for the duality product. Next we denote by $\|.\|_{W^{\prime}}$ the dual norm of $W$.

Let be given the following forms for any $u=\left(u_{1}, u_{2}\right), v=\left(v_{1}, v_{2}\right)$ in $V$ and $\mu \in W^{\prime}$ :

$$
\begin{gathered}
a(u, v)=\sum_{\ell=1}^{2} \int_{\Omega^{\ell}} \mathcal{A}_{\ell} \varepsilon\left(u_{\ell}\right): \varepsilon\left(v_{\ell}\right) d \Omega^{\ell}, \quad l(v)=\sum_{\ell=1}^{2} \int_{\Omega^{\ell}} f_{\ell} \cdot v_{\ell} d \Omega^{\ell}+\int_{\Gamma_{N}^{\ell}} F_{\ell} \cdot v_{\ell} d \Gamma^{\ell}, \\
b(\mu, v)=\int_{\Gamma_{C}} \mu\left[v_{N}\right] d \Gamma .
\end{gathered}
$$

The weak formulation of Problem (1)-(7) is to find $u \in V$ and $\lambda \in M$ such that

$$
\left\{\begin{array}{l}
a(u, v)-b(\lambda, v)=l(v), \quad \forall v \in V \\
b(\mu-\lambda, u) \geq 0, \quad \forall \mu \in M
\end{array}\right.
$$

The existence and uniqueness of $(u, \lambda)$ solution to (8) has been first stated in [1]. Moreover, the second argument $\lambda$ solution to (8) satisfies $\lambda=\sigma_{N}$. 


\section{The Local Average Contact (LAC) method}

\subsection{Definition}

Let $V_{\ell}^{h} \subset V_{\ell}$ be a family of finite dimensional vector spaces indexed by $h_{\ell}$ coming from a regular family $\mathcal{T}_{\ell}^{h}$ of triangulations, quadrangulations, tetrahedralizations or hexahedralizations of the domains $\Omega^{\ell}, \ell=1,2$ (see [30, $31,32])$. We assume that the boundary parts $\Gamma_{N}^{\ell}, \Gamma_{D}^{\ell}$ and $\Gamma_{C}^{\ell}$ are consistent with the discretizations of $\Omega^{\ell}$ and we set $\Gamma_{C}=\Gamma_{C}^{1} \cap \Gamma_{C}^{2}$. Note that we do not suppose that $\Gamma_{C}$ is consistent with one of the discretizations (in other words $\Gamma_{C}$ is neither a union of trace meshes coming from one nor from the other body), see Figure 1.

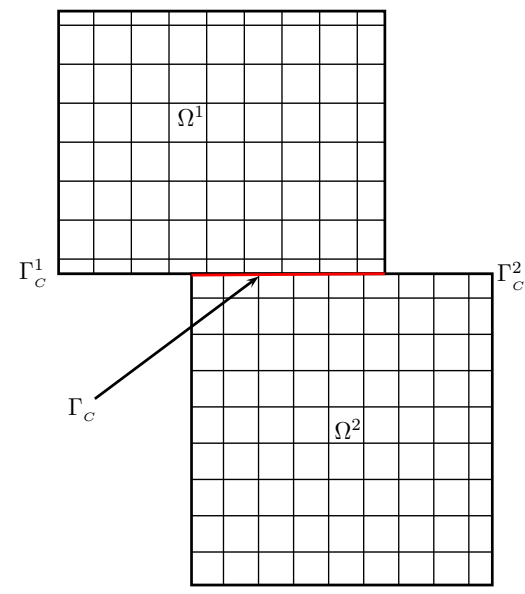

Figure 1: Problem set-up

The notation $h_{\ell}$ represents the largest diameter among all (closed) elements $\mathcal{T} \in \mathcal{T}_{\ell}^{h}$. We choose standard continuous and piecewise affine or quadratic functions, i.e.:

$$
V_{\ell}^{h}=\left\{v_{\ell}^{h} \in\left(C\left(\bar{\Omega}^{\ell}\right)\right)^{d}:\left.v_{\ell}^{h}\right|_{\mathcal{T}} \in P_{k}(\mathcal{T}), \forall \mathcal{T} \in \mathcal{T}_{\ell}^{h}, v_{\ell}^{h}=0 \text { on } \Gamma_{D}^{\ell}\right\}
$$

where $d=2,3$ and $k=1,2$. We set

$$
V^{h}=V_{1}^{h} \times V_{2}^{h} .
$$

We have to introduce the internal degree of freedom hypothesis which is needed to carry out the convergence analysis. 
Hypothesis 1. (internal d.o.f.) There exists a (d-1 dimensional) macromesh $T^{M}$ of $\Gamma_{C}^{1}$ (or of $\Gamma_{C}^{2}$ ) whose macro-elements are unions of elements of $\mathcal{T}_{1}^{h} \cap \Gamma_{C}^{1}$ (or of $\mathcal{T}_{2}^{h} \cap \Gamma_{C}^{2}$ ) such that for every macro-element $T^{m} \in T^{M}$, there exists (at least) a degree of freedom $x_{i}$ of $V_{\ell}^{h}$ such that $\operatorname{supp}\left(\phi_{i}\right) \subset T^{m}$, where $\phi_{i}$ is the trace of the basis function associated to $x_{i}$. Moreover there exists a constant $C$ such that the largest diameter of a the macro-element is lower than $C_{\ell}$ (this last requirement is made to avoid a too coarse macro-mesh).

From a theoretical point of view a macro-mesh satisfying Hypothesis 1 always exist if the mesh is fine enough (it suffices to gather the elements) but the practical construction of the macro-mesh is a key point of the LAC method in particular in the threedimensional case (see the implementation hereafter, e.g. section 4.4 and Figure 2).

From now on we suppose that $T^{M}$ is a macro-mesh of (e.g.) $\Gamma_{C}^{1}$ satisfying Hypothesis 1.

In the forthcoming definition of the LAC method we have also to take into account that $\Gamma_{C}=\Gamma_{C}^{1} \cap \Gamma_{C}^{2}$ does not coincide with a union of trace meshes of one or the other body. So we only consider the macro-elements which have a sufficiently large intersection with $\Gamma_{C}$ and we simply neglect the contact contribution of the macro-elements with a too small intersection. The size of the intersection is evaluated using the parameter $\varepsilon \in(0,1]$ in the forthcoming definition.

Definition 1. Let $\varepsilon>0$ be a fixed small parameter. Suppose that Hypothesis 1 holds. The macro-element $T^{m}$ is said to be admissible if

$$
\frac{\int_{T^{m} \cap \Gamma_{C}} \phi_{m^{\star}+1} d \Gamma}{\sqrt{\left|T^{m} \cap \Gamma_{C}\right|\left|T^{m}\right|}}=\overline{\phi_{m^{\star}+1}} \sqrt{\delta} \geq \varepsilon
$$

where $\phi_{m^{\star}+1}$ is the basis function associated to the internal degree of freedom, $\overline{\phi_{m^{\star}+1}}$ denotes its average over $T^{m} \cap \Gamma_{C}$ and $\delta=\left|T^{m} \cap \Gamma_{C}\right| /\left|T^{m}\right|$ is the ratio of the element $T^{m}$ belonging to $\Gamma_{C}$. We denote by $T_{a d}^{M}$ the set of admissible elements in $\Gamma_{C}^{1}$.

It is easy to see that any macro-element included into $\overline{\Gamma_{C}}$ (i.e. $\delta=1$ ) is admissible provided that $\varepsilon$ is smaller than a value depending only on $d$ and on choice of the finite element (e.g., $1 / 2$ for linear elements and $d=2,1 / 3$ for linear elements and $d=3$ ). Conversely any macro-element $T^{m}$ having an 
empty intersection with $\Gamma_{C}$ (i.e. $\left.\delta=0\right)$ is never admissible. In the remaining case (i.e., $T^{m}$ intersects partially $\Gamma_{C}$ ). $T^{m}$ is admissible if (roughly speaking) its intersection with $\Gamma_{C}$ is not too small.

Remark 1. If $T^{m}$ contains more than one internal d.o.f. then we fix one of them which satisfies $\left|T^{m} \cap \Gamma_{C}\right|>0$, which is denoted $x_{m^{\star}+1}$ and the other internal d.o.f. are handled as standard nodes.

Therefore the candidate contact area $\Gamma_{C}$ can be written as a disjoint union:

$$
\Gamma_{C}=\Gamma_{C}^{a d} \cup \Gamma_{C}^{\varepsilon}
$$

where $\Gamma_{C}^{a d}=\cup_{T^{m} \in T_{a d}^{M}}\left(T^{m} \cap \Gamma_{C}\right)$ denotes the set of admissible macro-elements $T^{m}$ intersected with $\Gamma_{C}$ and $\Gamma_{C}^{\varepsilon}$ stands for the remaining area.

We choose piecewise constant nonpositive Lagrange multipliers on $\Gamma_{C}$ which vanish on $\Gamma_{C}^{\varepsilon}$, i.e., in the convex cone $M^{h}$ :

$$
M^{h}=\left\{\mu^{h} \in X^{h}: \mu^{h} \leq 0 \text { on } \Gamma_{C}\right\}
$$

with

$$
X^{h}=\left\{\mu^{h} \in L^{2}\left(\Gamma_{C}\right): \mu^{h} \underset{T^{m} \cap \Gamma_{C}, T^{m} \in T^{M}}{\in P_{0}}\left(T_{C}^{m}\right),\left.\mu^{h}\right|_{\Gamma_{C}^{\varepsilon}}=0\right\} .
$$

Note that $X^{h} \subset W^{\prime}$ so that the bilinear form $b$ is well defined on $X^{h} \times V^{h}$. The discrete problem to be solved becomes: find $u^{h} \in V^{h}$ and $\lambda^{h} \in M^{h}$ such that

$$
\left\{\begin{array}{l}
a\left(u^{h}, v^{h}\right)-b\left(\lambda^{h}, v^{h}\right)=l\left(v^{h}\right), \forall v^{h} \in V^{h}, \\
b\left(\mu^{h}-\lambda^{h}, u^{h}\right) \geq 0, \forall \mu^{h} \in M^{h} .
\end{array}\right.
$$

The existence of a solution $\left(u^{h}, \lambda^{h}\right)$ of (9) as well as the uniqueness of $u^{h}$ follows from standard arguments (see, e.g., [1]). When $\Gamma_{C}$ is consistent with one of the discretizations (i.e., $\Gamma_{C}$ is a union of trace meshes coming from one or from the other body), which corresponds to the case $\Gamma_{C}=\Gamma_{C}^{a d}$ or equivalently $\Gamma_{C}^{\varepsilon}=\emptyset$, the uniqueness of $\lambda^{h}$ can be established (see $[33,29]$ ) as well as the existence of a constant $\beta^{h}$ such that

$$
\inf _{\mu^{h} \in X^{h}} \sup _{v^{h} \in V^{h}} \frac{b\left(\mu^{h}, v^{h}\right)}{\left\|\mu^{h}\right\|_{W^{\prime}}\left\|v^{h}\right\|} \geq \beta^{h}>0
$$

Again, in this case it can be proved that $\beta^{h}$ is independent of $h$, i.e., $\beta^{h}=\beta$ (see the details in $[33,29]$ ). 
When $\Gamma_{C}$ is not consistent with one of the discretizations, in order to prove the uniqueness of the solution $\left(u^{h}, \lambda^{h}\right)$ of (9) as well as the existence of $\beta^{h}$ in (10) we need to check that for any $\mu^{h} \in X^{h}$ we have the following implication $b\left(\mu^{h}, v^{h}\right)=0, \forall v^{h} \in V^{h} \Rightarrow \mu^{h}=0$. In the case of linear finite elements, we can use Lemma 3.1 in [33] or Lemma 1 in [29] and the result is obvious since all the basis fonctions are nonnegative everywhere. The dependence of $\beta^{h}$ on $h$ in the general case will be studied in the appendix.

Remark 2. An equivalent formulation: the discrete set of admissible displacements satisfying the average noninterpenetration conditions on the contact zone is given by

$$
K_{\varepsilon}^{h}=\left\{v^{h} \in V^{h}: \int_{T^{m} \cap \Gamma_{C}}\left[v_{N}^{h}\right] d \Gamma \leq 0, \quad \forall T^{m} \in T_{a d}^{M}\right\} .
$$

The corresponding discrete variational inequality is to find $u^{h} \in K_{\varepsilon}^{h}$ such that $a\left(u^{h}, v^{h}-u^{h}\right) \geq l\left(v^{h}-u^{h}\right), \forall v^{h} \in K_{\varepsilon}^{h}$. According to Stampacchia's Theorem, this inequality admits a unique solution and its solution $u^{h}$ coincides with the first argument of the solution to (9).

\subsection{Error estimates}

\subsubsection{An abstract error estimate}

We begin with a standard abstract error estimate.

Proposition 1. Let $(u, \lambda)$ be the solution of (8) and let $\left(u^{h}, \lambda^{h}\right)$ be the solution of (9). Then, there exists a positive constant $C$ independent of $h$ and $\varepsilon$ satisfying:

$$
\begin{aligned}
\left\|u-u^{h}\right\| \leq & C\left\{\left(1+\frac{1}{\beta^{h}}\right) \inf _{v^{h} \in V^{h}}\left\|u-v^{h}\right\|+\inf _{\mu^{h} \in X^{h}}\left\|\lambda-\mu^{h}\right\|_{W^{\prime}}\right. \\
& \left.+\left(\max \left(0,-b\left(u^{h}, \lambda\right)\right)\right)^{1 / 2}\right\} \\
\left\|\lambda-\lambda^{h}\right\|_{W^{\prime}} \leq & C\left(1+\frac{1}{\beta^{h}}\right)\left(\left\|u-u^{h}\right\|+\inf _{\mu^{h} \in X^{h}}\left\|\lambda-\mu^{h}\right\|_{W^{\prime}}\right) .
\end{aligned}
$$


Proof. The proof is classical, we write it here only to show the dependence of the inf-sup constant $\beta^{h}$. Let $v^{h} \in V^{h}$. According to (8) and (9), we have

$$
\begin{aligned}
a\left(u-u^{h}, u-u^{h}\right) & =a\left(u-u^{h}, u-v^{h}\right)+a\left(u, v^{h}-u^{h}\right)-a\left(u^{h}, v^{h}-u^{h}\right) \\
& =a\left(u-u^{h}, u-v^{h}\right)+b\left(\lambda, v^{h}-u^{h}\right)-b\left(\lambda^{h}, v^{h}-u^{h}\right) \\
& =a\left(u-u^{h}, u-v^{h}\right)+b\left(\lambda-\lambda^{h}, v^{h}-u\right)+b\left(\lambda-\lambda^{h}, u-u^{h}\right) .
\end{aligned}
$$

Besides, we have $b(\lambda, u)=0$. Similarly, (9) leads to $b\left(\lambda^{h}, u^{h}\right)=0$. Therefore

$a\left(u-u^{h}, u-u^{h}\right)=a\left(u-u^{h}, u-v^{h}\right)-b\left(\lambda-\lambda^{h}, u-v^{h}\right)-b\left(\lambda, u^{h}\right)-b\left(\lambda^{h}, u\right)$.

Denoting by $\alpha$ the ellipticity constant of $a(.,$.$) on V$, by $M$ the continuity constant of $a(.,$.$) on V$ and using the trace theorem, we obtain:

$$
\begin{aligned}
\alpha\left\|u-u^{h}\right\|^{2} \leq & M\left\|u-u^{h}\right\|\left\|u-v^{h}\right\|+C\left\|\lambda-\lambda^{h}\right\|_{W^{\prime}}\left\|u-v^{h}\right\| \\
& -b\left(\lambda, u^{h}\right)-b\left(\lambda^{h}, u\right) .
\end{aligned}
$$

Now, let us consider problem (8). The inclusion $V^{h} \subset V$ implies

$$
a\left(u, v^{h}\right)-b\left(\lambda, v^{h}\right)=l\left(v^{h}\right), \quad \forall v^{h} \in V^{h} .
$$

The latter equality together with (9) yields

$$
a\left(u-u^{h}, v^{h}\right)-b\left(\lambda-\lambda^{h}, v^{h}\right)=0, \quad \forall v^{h} \in V^{h} .
$$

Inserting $\mu^{h} \in X^{h}$, using the continuity of $a(.,$.$) as well as the trace$ theorem gives

$$
\begin{aligned}
b\left(\lambda^{h}-\mu^{h}, v^{h}\right) & =-a\left(u-u^{h}, v^{h}\right)+b\left(\lambda-\mu^{h}, v^{h}\right) \\
& \leq M\left\|u-u^{h}\right\|\left\|v^{h}\right\|+C\left\|\lambda-\mu^{h}\right\|_{W^{\prime}}\left\|v^{h}\right\|, \forall \mu^{h} \in X^{h}, \forall v^{h} \in V^{h} .
\end{aligned}
$$

The inf-sup condition and the previous bound allow us to write

$$
\beta^{h}\left\|\lambda^{h}-\mu^{h}\right\|_{W^{\prime}} \leq \sup _{v^{h} \in V^{h}} \frac{b\left(\lambda^{h}-\mu^{h}, v^{h}\right)}{\left\|v^{h}\right\|} \leq M\left\|u-u^{h}\right\|+C\left\|\lambda-\mu^{h}\right\|_{W^{\prime}},
$$

for any $\mu^{h} \in X^{h}$. 
Since

$$
\left\|\lambda-\lambda^{h}\right\|_{W^{\prime}} \leq\left\|\lambda-\mu^{h}\right\|_{W^{\prime}}+\left\|\mu^{h}-\lambda^{h}\right\|_{W^{\prime}}, \quad \forall \mu^{h} \in X^{h},
$$

we finally come to the conclusion that there exists $C>0$ such that

$$
\left\|\lambda-\lambda^{h}\right\|_{W^{\prime}} \leq C\left(1+\frac{1}{\beta^{h}}\right)\left(\left\|u-u^{h}\right\|+\inf _{\mu^{h} \in X^{h}}\left\|\lambda-\mu^{h}\right\|_{W^{\prime}}\right) .
$$

Putting together (12) and (11) and using Young inequality gives any $v^{h} \in V^{h}$

$$
\begin{aligned}
\alpha\left\|u-u^{h}\right\|^{2} \leq C & \left\{\left(1+\frac{1}{\beta^{h}}\right)^{2} \inf _{v^{h} \in V^{h}}\left\|u-v^{h}\right\|^{2}+\inf _{\mu^{h} \in X^{h}}\left\|\lambda-\mu^{h}\right\|_{W^{\prime}}^{2}\right\} \\
& -b\left(\lambda, u^{h}\right)-b\left(\lambda^{h}, u\right),
\end{aligned}
$$

and, since $b\left(\lambda^{h}, u\right) \geq 0$, we conclude that

$$
\begin{aligned}
\alpha\left\|u-u^{h}\right\| \leq C & \left\{\left(1+\frac{1}{\beta^{h}}\right) \inf _{v^{h} \in V^{h}}\left\|u-v^{h}\right\|+\inf _{\mu^{h} \in X^{h}}\left\|\lambda-\mu^{h}\right\|_{W^{\prime}}\right\} \\
& +\left(\max \left(0,-b\left(\lambda, u^{h}\right)\right)\right)^{1 / 2} .
\end{aligned}
$$

\subsubsection{Error estimate when $\Gamma_{C}$ is consistent with the discretization}

We first give an error estimate when $\Gamma_{C}$ is consistent with the discretization either of $\Omega^{1}$ (as in the next theorem) or of $\Omega^{2}$. Of course, the meshes of $\Omega^{1}$ and $\Omega^{2}$ are not supposed to fit together on $\Gamma_{C}$, so there are nonmatching meshes on the contact area.

Theorem 1. Let $d=2,3$ and $k=1,2$. Suppose that $\Gamma_{C}$ is consistent with the discretization of e.g. $\Omega^{1}$. Let $(u, \lambda)$ and $\left(u^{h}, \lambda^{h}\right)$ be the solutions to the continuous and to the discrete problems (8) and (9) respectively where the Hypothesis 1 is used to build the Lagrange multiplier space. Assume that $u \in\left(H^{\tau}\left(\Omega^{1}\right)\right)^{d} \times\left(H^{\tau}\left(\Omega^{2}\right)\right)^{d}$ with $3 / 2<\tau \leq \min (k+1,5 / 2)$. Then, there exists a constant $C>0$ independent of $h=\max \left(h_{1}, h_{2}\right)$ and $u$ such that

$$
\left\|u-u^{h}\right\|+\left\|\lambda-\lambda^{h}\right\|_{W^{\prime}} \leq C h^{\tau-1}\|u\|_{\tau, \Omega} .
$$

Proof. In this case all the macro-elements are admissible since $\Gamma_{C}$ is the union of (entire) macro-elements, the inf-sup constant $\beta_{h}$ does not depend on $h$ (see [29] or the appendix) and it suffices to choose in Proposition 1 $v^{h}=\left(I^{h} u_{1}, I^{h} u_{2}\right)$ where $I^{h}$ is the Lagrange interpolation operator of degree $k, \mu^{h}=\bar{\pi}_{1}^{h} \lambda$ where $\bar{\pi}_{1}^{h}$ is the $L^{2}$-projection operator onto $X^{h}\left(\Gamma_{C}^{\varepsilon}=\emptyset\right)$ which lead to standard optimal error estimates. The remaining error estimate on $b\left(\lambda, u^{h}\right)$ can be found in [29] and uses the techniques developed in [34]. 


\subsubsection{Error estimate when $\Gamma_{C}$ is not consistent with the discretization}

The forthcoming theorem is concerned with the general case (nonmatching meshes and geometrical nonconforming case), i.e., when $\Gamma_{C}$ is consistent with none of both discretizations of $\Omega^{\ell}$. Actually, for technical reasons, we are only able to achieve the analysis for linear finite elements.

Theorem 2. Let $d=2,3$ and $k=1$. Suppose that $\Gamma_{C}$ is consistent with none discretization of $\Omega^{\ell}, \ell=1,2$. Let $(u, \lambda)$ and $\left(u^{h}, \lambda^{h}\right)$ be the solutions to the continuous and to the discrete problems (8) and (9) respectively where the Hypothesis 1 is used to build the Lagrange multiplier space. Assume that $u \in\left(H^{\tau}\left(\Omega^{1}\right)\right)^{d} \times\left(H^{\tau}\left(\Omega^{2}\right)\right)^{d}$ with $3 / 2<\tau \leq 2$. Then, there exists constants $C, C(\eta)>0$ independent of $h=\max \left(h_{1}, h_{2}\right)$ and $u$ such that when $d=2$ :

$$
\left\|u-u^{h}\right\|+\left\|\lambda-\lambda^{h}\right\|_{W^{\prime}} \leq\left\{\begin{array}{l}
C h^{\tau-1} \sqrt{-\ln (h)}\|u\|_{\tau, \Omega}, \quad 3 / 2<\tau<2, \\
C(\eta) h^{1-\eta}\|u\|_{2, \Omega}, \forall \eta>0, \quad \tau=2,
\end{array}\right.
$$

and when $d=3$ :

$$
\left\|u-u^{h}\right\|+h^{\frac{3(\tau-1)}{2(\tau+2)}}\left\|\lambda-\lambda^{h}\right\|_{W^{\prime}} \leq C h^{(\tau-1) \frac{2 \tau+1}{2 \tau+4}}\|u\|_{\tau, \Omega} \quad 3 / 2<\tau \leq 2 .
$$

We note that the estimates are quasi-optimal in 2D. On the contrary we are not able (for technical reasons) to obtain the same quasi-optimal convergence rates in 3D and we only prove convergence with some lower rates. As far as we know these estimates are the first ones in the geometrical nonconforming case. Note that there are other techniques which could maybe be adapted to our problem such as the ones issued from fictituous domains or extended finite element methods (see, e.g., [35, 36]) already applied to contact problems in a different context.

Proof. We use again Proposition 1 and we have to bound two norm terms and $-b\left(\lambda, u^{h}\right)$. As in Theorem 1 , we choose $v^{h}=\left(I^{h} u_{1}, I^{h} u_{2}\right)$ and $\mu^{h}=\bar{\pi}_{1}^{h} \lambda$. Obviously $\left\|u-v^{h}\right\| \leq C h^{\tau-1}\|u\|_{\tau, \Omega}$.

We have $-b\left(\lambda, u^{h}\right)=-\int_{\Gamma_{C}^{a d}} \lambda\left[u_{N}^{h}\right] d \Gamma-\int_{\Gamma_{C}^{\varepsilon}} \lambda\left[u_{N}^{h}\right] d \Gamma$. By using standard techniques as in [29], it is easy to show that the first integral term yields:

$$
-\int_{\Gamma_{C}^{a d}} \lambda\left[u_{N}^{h}\right] d \Gamma \leq C h^{2(\tau-1)}\|u\|_{\tau, \Omega}^{2}+\frac{\alpha}{2}\left\|u-u^{h}\right\|^{2} .
$$

The second integral term coming from $-b\left(\lambda, u^{h}\right)$ can be bounded using $\lambda u_{N}=$ 0, generalized Hölder inequalities, Sobolev embeddings and the trace theo- 
rem: for $d=2$ and $1<p<2$ (see [37, 38]):

$$
\begin{aligned}
-\int_{\Gamma_{C}^{\varepsilon}} \lambda\left[u_{N}^{h}\right] d \Gamma & =\int_{\Gamma_{C}^{\varepsilon}} \lambda\left[\left(u-u^{h}\right)_{N}\right] d \Gamma \\
& \leq\|\lambda\|_{L^{p}\left(\Gamma_{C}^{\varepsilon}\right)}\left\|\left[\left(u-u^{h}\right)_{N}\right]\right\|_{L^{p /(p-1)}\left(\Gamma_{C}\right)} \\
& \leq C \sqrt{\frac{p}{p-1}}\|\lambda\|_{L^{1 /(2-\tau)}\left(\Gamma_{C}^{\varepsilon}\right)}\left|\Gamma_{C}^{\varepsilon}\right|^{\tau-2+1 / p}\left\|\left[\left(u-u^{h}\right)_{N}\right]\right\|_{1 / 2, \Gamma_{C}} \\
& \leq C \sqrt{\frac{p}{p-1}}\|\lambda\|_{\tau-3 / 2, \Gamma_{C}}\left|\Gamma_{C}^{\varepsilon}\right|^{\tau-2+1 / p}\left\|u-u^{h}\right\|
\end{aligned}
$$

for $3 / 2<\tau<2$ and where $\left|\Gamma_{C}^{\varepsilon}\right|$ denotes the onedimensional measure of $\Gamma_{C}^{\varepsilon}$. Choosing $p=\ln \left|\Gamma_{C}^{\varepsilon}\right| /\left(1+\ln \left|\Gamma_{C}^{\varepsilon}\right|\right)$ gives

$$
-\int_{\Gamma_{C}^{\varepsilon}} \lambda\left[u_{N}^{h}\right] d \Gamma \leq C\left(-\ln \left|\Gamma_{C}^{\varepsilon}\right|\right)^{1 / 2}\|\lambda\|_{\tau-3 / 2, \Gamma_{C}}\left|\Gamma_{C}^{\varepsilon}\right|^{\tau-1}\left\|u-u^{h}\right\| .
$$

When $d=3$, the bound is obtained in a similar (and simpler) way:

$$
\begin{aligned}
-\int_{\Gamma_{C}^{\varepsilon}} \lambda\left[u_{N}^{h}\right] d \Gamma & \leq\|\lambda\|_{L^{4 / 3}\left(\Gamma_{C}^{\varepsilon}\right)}\left\|\left[\left(u-u^{h}\right)_{N}\right]\right\|_{L^{4}\left(\Gamma_{C}\right)} \\
& \leq C\|\lambda\|_{L^{4 /(5-2 \tau)}\left(\Gamma_{C}^{\varepsilon}\right)}\left\|\left[\left(u-u^{h}\right)_{N}\right]\right\|_{1 / 2, \Gamma_{C}}\left|\Gamma_{C}^{\varepsilon}\right|^{\frac{\tau-1}{2}} \\
& \leq C\|\lambda\|_{\tau-3 / 2, \Gamma_{C}}\left|\Gamma_{C}^{\varepsilon}\right|^{\frac{\tau-1}{2}}\left\|u-u^{h}\right\|,
\end{aligned}
$$

for $3 / 2<\tau<5 / 2$.

The bound of the second norm term $\left\|\lambda-\bar{\pi}_{1}^{h} \lambda\right\|_{W^{\prime}}$ in Proposition 1 needs an additional calculus since the functions of $X^{h}$ (here $\bar{\pi}_{1}^{h} \lambda$ ) vanish on $\Gamma_{C}^{\varepsilon}$. So

$$
\begin{aligned}
\left\|\lambda-\bar{\pi}_{1}^{h} \lambda\right\|_{W^{\prime}} & =\sup _{\psi \in W} \frac{\int_{\Gamma_{C}}\left(\lambda-\bar{\pi}_{1}^{h} \lambda\right) \psi d \Gamma}{\|\psi\|_{W}} \\
& \leq \sup _{\psi \in W} \frac{\int_{\Gamma_{C}^{a d}}\left(\lambda-\bar{\pi}_{1}^{h} \lambda\right) \psi d \Gamma}{\|\psi\|_{W}}+\sup _{\psi \in W} \frac{\int_{\Gamma_{C}^{\varepsilon}} \lambda \psi d \Gamma}{\|\psi\|_{W}} .
\end{aligned}
$$

The first supremum is optimally bounded in a standard way (using the approximation properties of $\bar{\pi}_{1}^{h}$, see, e.g. $\left.[33,34,29]\right)$ by $C h^{\tau-1}\|u\|_{\tau, \Omega}$. The second supremum comes from the geometrical nonconformity and is bounded 
exactly as in (14), (15) by changing $u-u^{h}$ with $\psi\left(\right.$ and $\left\|\left[\left(u-u^{h}\right)_{N}\right]\right\|_{1 / 2, \Gamma_{C}}$ with $\left.\|\psi\|_{W}\right)$.

According to the appendix, we can choose the inf-sup constant $\beta_{h}=C \varepsilon$. From the previous bounds and Proposition 1 , we get when $d=2$ and $3 / 2<$ $\tau<2$ :

$$
\left\|u-u^{h}\right\|+\varepsilon\left\|\lambda-\lambda^{h}\right\|_{W^{\prime}} \leq C\left(\varepsilon^{-1} h^{\tau-1}+\left|\Gamma_{C}^{\varepsilon}\right|^{\tau-1}\left(-\ln \left|\Gamma_{C}^{\varepsilon}\right|\right)^{1 / 2}\right)\|u\|_{\tau, \Omega} .
$$

It is easy to show that the measure of $\Gamma_{C}^{\varepsilon}$ is lower than $C \varepsilon^{2 / 3} h$. Therefore, fixing $\varepsilon$ small (and independent of $h$ ) we get the estimate:

$$
\left\|u-u^{h}\right\|+\left\|\lambda-\lambda^{h}\right\|_{W^{\prime}} \leq C(\varepsilon) h^{\tau-1} \sqrt{-\ln (h)}\|u\|_{\tau, \Omega}
$$

when the constant $\varepsilon$ is chosen independent of $h$. In the case $\tau=2$, estimate (13) does not hold: it suffices to see that $u$ also lies in $H^{2-\eta / 2}$ for any positive $\eta$, to use the previous bounds with $\tau=2-\eta / 2$ together with the asymptotical estimate $\sqrt{-\ln (h)} \leq C h^{-\eta / 2}$. Note that the bounds in the 2D case are quasioptimal.

When $d=3$ and $3 / 2<\tau \leq 2$ we use (15) and get the bound

$$
\left\|u-u^{h}\right\|+\varepsilon\left\|\lambda-\lambda^{h}\right\|_{W^{\prime}} \leq C\left(\varepsilon^{-1} h^{\tau-1}\|u\|_{\tau, \Omega}+\|\lambda\|_{\tau-3 / 2, \Gamma_{C}}\left|\Gamma_{C}^{\varepsilon}\right|^{(\tau-1) / 2}\right) .
$$

Given a nonadmissible triangular or quadrangular macro-element $T$, we want to bound the area of $T \cap \Gamma_{C}$ (which is a triangle or a quadrangle, since the intersection is supposed small and which intersects two or three edges of $T$ ). Let $\delta_{1} h$ and $\delta_{2} h$ be the lenghts of the two largest edges of $T \cap \Gamma_{C}$ included in the edges of $T$. It is easy to check that $\delta$ (resp. $\overline{\phi_{m^{\star}+1}}$ ) (see Definition 1 ) are equivalent to (up to constants independent of $h) \delta_{1} \delta_{2}\left(\operatorname{resp} \cdot \min \left(\delta_{1}, \delta_{2}\right)\right.$ ). Since the element is nonadmissible we have $\min ^{2}\left(\delta_{1}, \delta_{2}\right) \delta_{1} \delta_{2} \leq C \varepsilon^{2}$ hence $\delta_{1}^{3} \delta_{2}^{3} \leq C \varepsilon^{2}$ (note that this estimate is very rough) which implies that the area of $T \cap \Gamma_{C}$ is bounded by $C h^{2} \varepsilon^{2 / 3}$ and finally $\left|\Gamma_{C}^{\varepsilon}\right| \leq C h \varepsilon^{2 / 3}$. Therefore

$$
\left\|u-u^{h}\right\|+\varepsilon\left\|\lambda-\lambda^{h}\right\|_{W^{\prime}} \leq C\left(\varepsilon^{-1} h^{\tau-1}+\varepsilon^{(\tau-1) / 3} h^{(\tau-1) / 2}\right)\|u\|_{\tau, \Omega} .
$$

This suboptimal rate gives for the particular choice of $\varepsilon=h^{\frac{3(\tau-1)}{2(\tau+2)}}$ a bound of $h^{(\tau-1) \frac{2 \tau+1}{2 \tau+4}}$. 


\section{Implementation of method}

\subsection{Specifications}

To implement a new contact method in a standard finite element software (here [39]), we impose three constraints:

1. No use of non-local operators as much as possible and keep the independence of elementary quantities (vector, matrix) before assembly;

2. Possibility of using this method without changes of the most suitable hypotheses in the model (choice of elements, choice of material behaviour, etc...);

3. Possibility of using standard linear solvers for contact problems, in particular solvers which are now mostly parallelized on parallel computers.

\subsection{Comparing LAC method with other methods}

Several methods, based on mortar methods can been used. Any of the methods use $L^{2}$-projections on well chosen spaces, named here mortar spaces. When using linear and bilinear finite elements in 3D (4-node tetrahedra and 8-node hexahedra) the contact surface is made of triangles and quadrangles. There are usually three choices of mortar spaces, i.e.:

- " $P_{k-1}$ mortar": the space is defined by constant approximations in $P_{0}$ or $Q_{0}$ on the slave surface mesh, see $[40,41]$ for instance. This method needs a stabilization process.

- "Standard mortar": the space is directly built on the slave space discretization, see $[14,3,23,5]$.

- "Dual mortar": the space is the dual of the slave space discretization, see $[42,43]$.

When using three dimensional quadratic finite elements (10-node tetrahedra, 20 and 27-node hexahedra), the contact surface is made of 6-node triangles or 8 and 9-node quadrangles. Correct mortar space is more difficult to implement, and for some choices, we can obtain non-physical gaps between the surfaces. Two solutions were proposed:

- " $P_{k-1}$ mortar": the space is defined using a linear approximation $P_{1}$ or $Q_{1}$ on the slave surface mesh, [41, 24]. 
- "Dual quadratic mortar": the space is the dual of the slave space discretization, [43, 22]. It requires numerical basis functions that have to be computed (there are not defined by analytical functions) except for the easiest case of 27-node hexahedra.

After the choice of the correct mortar space, we have to define both the formulation and the algorithm to solve the contact problem. The "dual" and "dual quadratic" methods allow local static condensation of contact unknowns (Lagrange multipliers) thanks to the identity coupling matrix. The "standard" and " $P_{k-1}$ mortar" spaces use analytical basis functions. However, these methods need special adaptation of software to be implemented efficiently: the "standard" method $[12,13,23,24]$ is non local and as already mentioned the "dual" method $[42,22]$ uses basis functions which need to be numerically computed.

\subsection{Problem to solve}

We recall that the discrete problem to be solved is (see (9)): find $u^{h} \in V^{h}$ and $\lambda^{h} \in M^{h}$ such that

$$
\left\{\begin{array}{l}
a\left(u^{h}, v^{h}\right)-b\left(\lambda^{h}, v^{h}\right)=l\left(v^{h}\right), \forall v^{h} \in V^{h}, \\
b\left(\mu^{h}-\lambda^{h}, u^{h}\right) \geq 0, \forall \mu^{h} \in M^{h},
\end{array}\right.
$$

with

$$
M^{h}=\left\{\mu^{h} \in L^{2}\left(\Gamma_{C}\right): \mu_{\mid T^{m}}^{h} \in P_{0}\left(T^{m}\right), \forall T^{m} \in T^{M}, \mu^{h} \leq 0 \text { on } \Gamma_{C}\right\} .
$$

This " $P_{0}$ mortar" has been automatically stabilized thanks to the convenient definition of $T^{M}$ (hypothesis 1). We define the space $\mathcal{M}_{n, k}(\mathbb{R})$ of rectangular real matrices with $n$ lines and $k$ columns and $\mathcal{M}_{k}(\mathbb{R})=\mathcal{M}_{k, k}(\mathbb{R})$. The matrix $C^{S} \in \mathcal{M}_{n, k}(\mathbb{R})$ and $C^{M} \in \mathcal{M}_{m, k}(\mathbb{R})$ are defined by:

$$
C_{i j}^{S}=\int_{T_{j}} \phi_{i_{N}}^{S} d \Gamma, C_{i j}^{M}=\int_{T_{j}} \phi_{i_{N}}^{M} d \Gamma,
$$

and the matrix $M^{L A C} \in \mathcal{M}_{k}(\mathbb{R})$ is:

$$
M_{i j}^{L A C}=\delta_{i j}\left|T^{i}\right|,
$$

where $\phi^{S}\left(\right.$ resp. $\left.\phi^{M}\right)$ denotes the basis function of the finite element method on the slave (resp. master) surface and $T^{i}$ (resp. $\left.\left|T^{i}\right|\right)$ is the support (resp. 
the measure of the support) of the basis function $P_{0}\left(T^{M}\right)$ (on macro-mesh). Writing the displacements $U$ as follows: $U=\left(U^{N}, U^{M}, U^{S}\right)\left(U^{N}\right.$ involves the d.o.f. inside the bodies, $U^{M}$ and $U^{S}$ contain the d.o.f. of the master and slave parts) and denotong by $\Lambda$ the vector of Lagrange multipliers, the matrix formulation of (9) becomes:

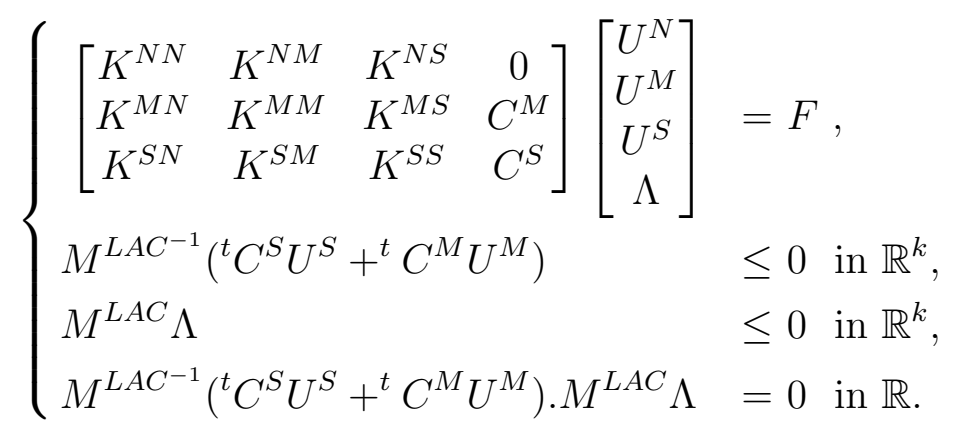

Note that $M^{L A C}$ is a diagonal and positive-definite matrix, we can write (16) in this equivalent form:

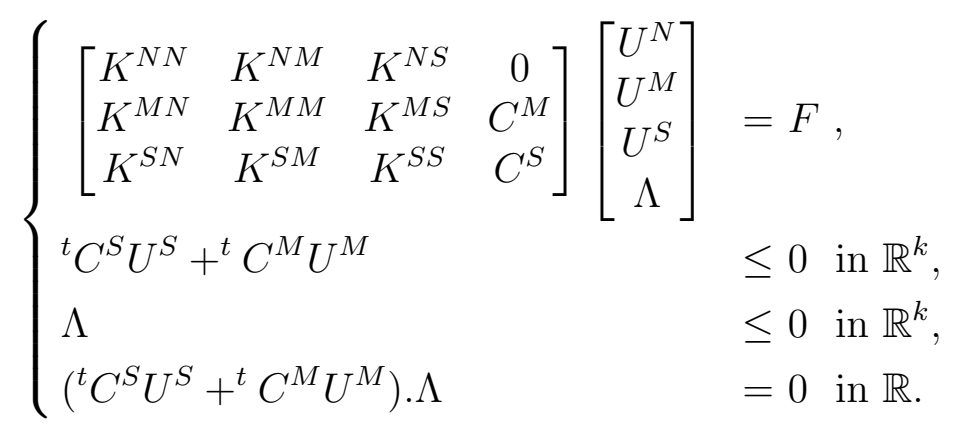

\subsection{Building the macro-mesh $T^{M}$}

From a practical point of view, an essential question is "how can Hypothesis 1 be fulfilled?"

1. In the twodimensional case with linear elements it suffices to gather the elements to obtain a macro mesh $T^{M}$;

2. In the twodimensional case with quadratic elements, the trace mesh already satisfies Hypothesis 1;

3. In the threedimensional case a refinement strategy is adopted. The strategy is depicted on Figure 2. For 27-node hexahedra, local refinement is not required (as in the 2D quadratic case) since there already exists a node located at the center of the contact face. This refinement 


Refinement for 4-node tetrahedron

Figure 2: Refinement of the first layer of elements for contact

strategy is local and only concerns the elements which have a face on the contact area.

One can either choose to refine the mesh of one or of the other body. Moreover the above-mentioned refinement does not affect the regularity and the quasi-uniformness properties of the meshes.

Remark 3. 1. This strategy could be easily extended to other elements (pyramids or prisms for instance);

2. The local refinement for an hexahedra is not unique, see Figure 2.

3. The preparation of the contact surface (see previous item in the remark) is achieved at the beginning of the computation and then never changes. Of course it could been "hidden" to code users. 


\subsection{General algorithm}

We choose a full-Newton algorithm based on a single Newton step for all non-linearities: contact and other non-linear mechanical behaviours (large strains, plasticity, etc...). The general algorithm is based on a Generalized Newton Method [44]. The contact status are computed with the active-set method strategy [45]. We have:

$$
\left[\begin{array}{l}
U \\
\Lambda
\end{array}\right]=\left[\begin{array}{c}
U^{N} \\
U^{M} \\
U^{S} \\
\Lambda
\end{array}\right], \quad K=\left[\begin{array}{ccc}
K^{N N} & K^{N M} & K^{N S} \\
K^{M N} & K^{M M} & K^{M S} \\
K^{S N} & K^{S M} & K^{S S}
\end{array}\right], \quad C=\left[\begin{array}{c}
0 \\
C^{M} \\
C^{S}
\end{array}\right]
$$

Given an initial gap $g_{0}$, the average initial gap $\overline{G_{0}}$ on the contact zone is:

$$
\overline{G_{0}}=\int_{T^{m} \cap \Gamma_{C}} g_{0} d \Gamma .
$$

The problem (17) is equivalent to (when taking into account the initial gap):

$$
\begin{cases}{\left[\begin{array}{ll}
K & C
\end{array}\right] \times\left[\begin{array}{l}
U \\
\Lambda
\end{array}\right]} & =F, \\
& \leq \overline{G_{0}} \text { in } \mathbb{R}^{k}, \\
{ }^{t} C U & \leq 0 \text { in } \mathbb{R}^{k}, \\
\Lambda & =0 \text { in } \mathbb{R} .\end{cases}
$$

We define the function $A$ :

$$
A(U, \Lambda)=\Lambda+\max \left(0, \rho\left(C U-\overline{G_{0}}\right)-\Lambda\right), \forall \rho>0 .
$$

Therefore (18) is equivalent to:

$$
\left\{\begin{aligned}
{\left[\begin{array}{ll}
K & C
\end{array}\right] \times\left[\begin{array}{l}
U \\
\Lambda
\end{array}\right] } & =F \\
A(U, \Lambda) & =0
\end{aligned}\right.
$$

We define $S\left(\Lambda^{i}\right)$ as the status of contact for each Lagrange multiplier indexed by $i$. To solve the non-linearity coming from the contact, we have to establish the active-set i.e., $S\left(\Lambda^{i}\right)=1$ and the non-active set $S\left(\Lambda^{i}\right)=0$. Finally we obtain the algorithm described hereafter. 


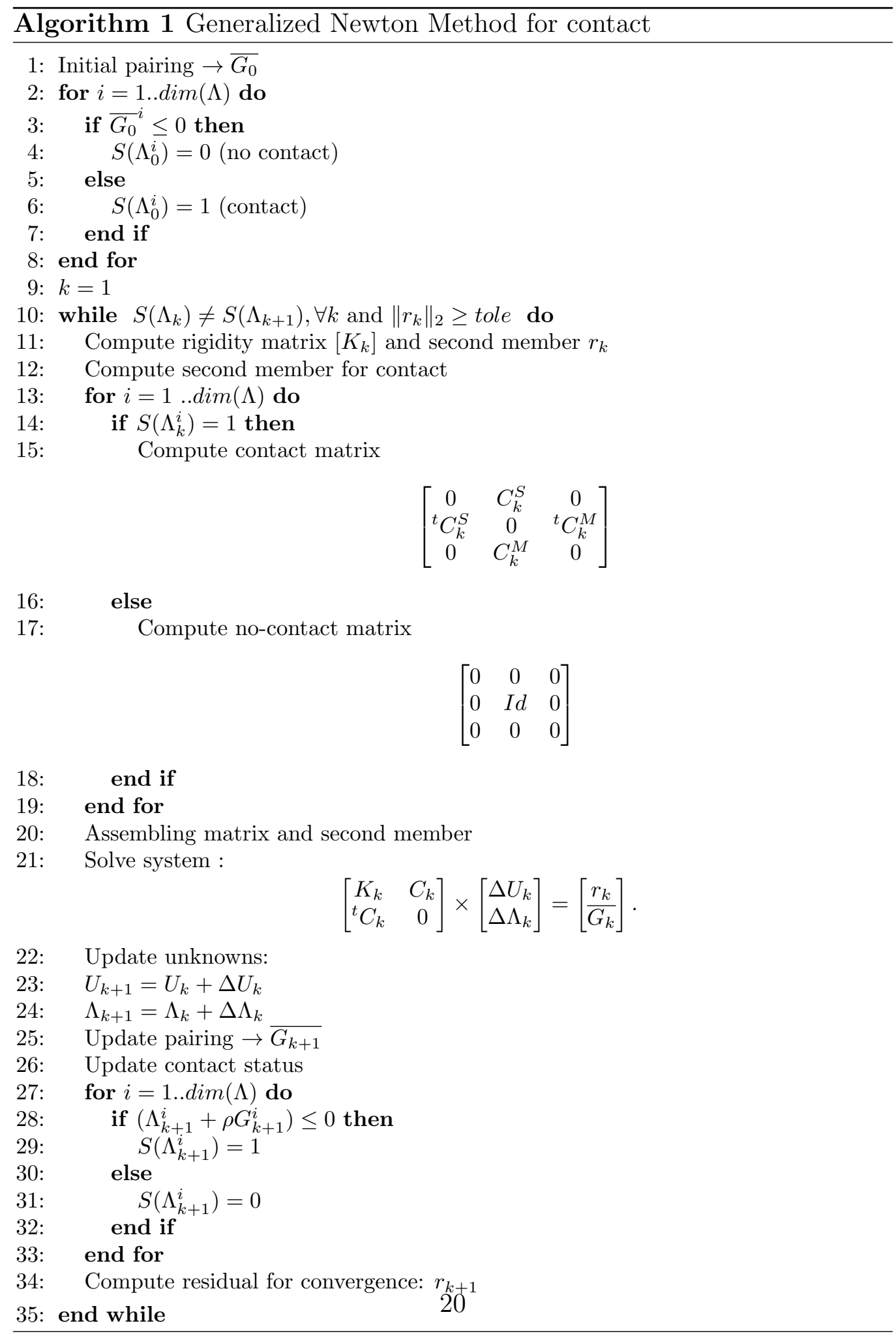




\subsection{Pairing algorithm}

For the pairing, we propose to adapt the PANG algorithm in [46] to the case of mortar contact. This algorithm is of linear complexity but it is not robust in the contact case. We change it to find all good initial contact pairs even with non-convex slave contact zone. The main principles of pairing algorithm are:

1. A robust and rapid algorithm to find all contact pair;

2. A Newton algorithm with line search to find the projection of a node on another element;

3. Using a reference space instead of a real space when computing the projection;

4. Using PANG's algorithm.

To evaluate several integrals we use a simple tesselation of the mortar contact zone.

\section{Numerical experiments}

In this section, we will analyze the behavior of the method facing some simple problems in two and three space dimensions. We consider the Taylor patch test (see [47]), the contact problems of two self included disks/spheres submitted to an internal pressure. Then we will confirm the theoretical convergence rate using the manufactured solution process. The last part of this section will be dedicated to a more realistic and complex contact problem, the crush of an hyperelastic half-ring on a hyperelastic foundation in the 3D case which illustrates also a geometrical nonconforming case (see $[48,49,27])$.

\subsection{Taylor patch test}

We consider a structure which consists of two identical cubes (or squares in 2D) of edge length $50 \mathrm{~mm}$. The material characteristics are: a Young modulus $E=2000 M P a$ and a Poisson ratio $\nu=0.3$. We set the following boundary conditions (in $3 \mathrm{D}$ ): symmetries hold on the lower part and on the $\overrightarrow{O x} \overrightarrow{O z}$ and $\overrightarrow{O y} \overrightarrow{O z}$ faces, a pressure $p=25 M P a$ is applied at the top of the structure. The expected results are a constant contact pressure and a uniform vertical Cauchy stress component equal to $25 \mathrm{MPa}$. The numerical results obtained for linear nonmatching meshes using 4-node quadrangles in $2 \mathrm{D}$ and 
8-node hexahedra in 3D with the LAC condition are depicted in Figures 3 and 4 .
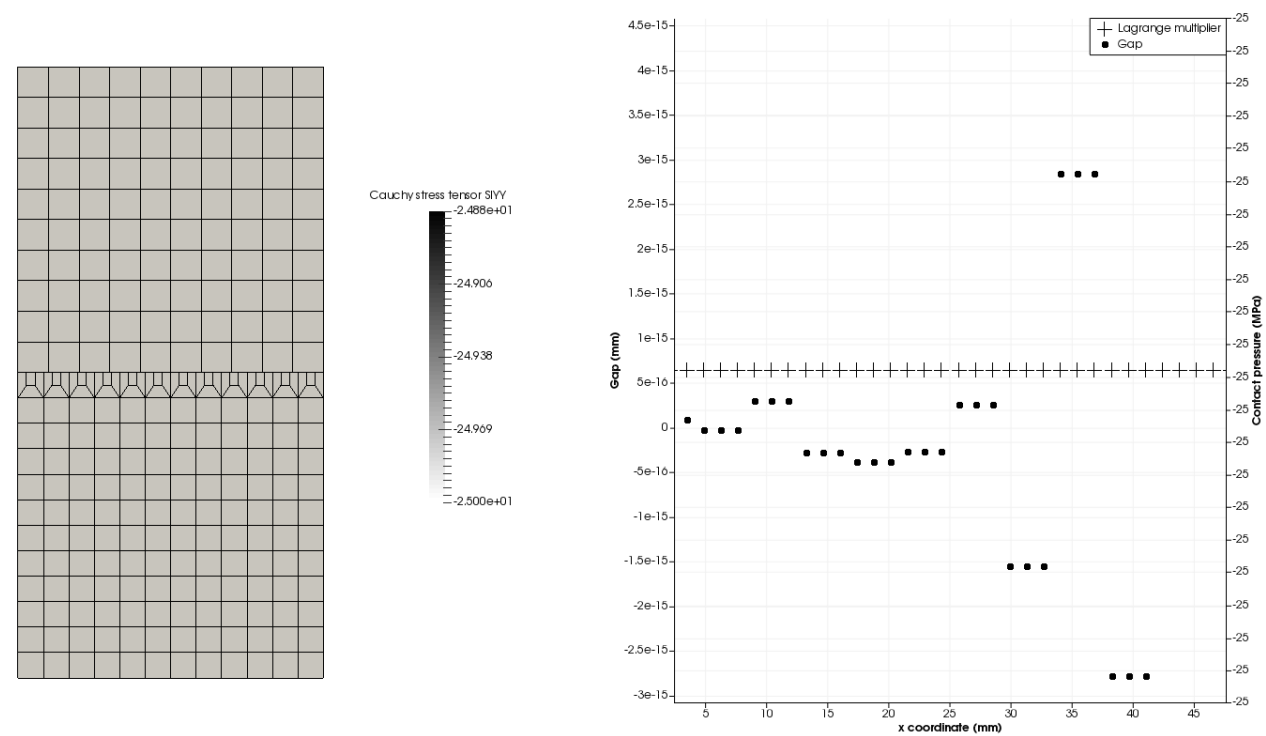

Figure 3: Numerical results obtained with the LAC condition in 2D when considering 4-node quadrangles: on the left $\sigma_{y y}$, on the right Lagrange multipliers and gap on $\Gamma_{C}$. 

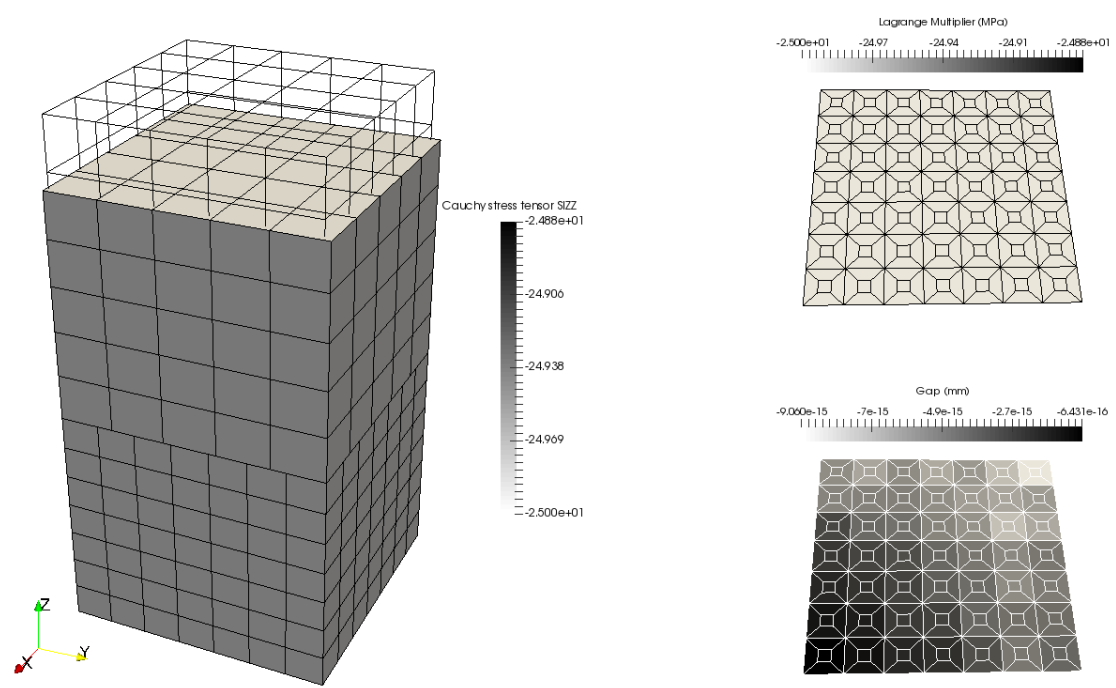

Figure 4: Numerical results obtained with the LAC condition in 3D when considering 8-node hexahedra: on the left $\sigma_{z z}$ on the deformed shape (scale factor 10), on the right Lagrange multipliers and gap on $\Gamma_{C}$.

This test case is successfully passed by the LAC method for all the considered linear elements. We see in Tables 1 and 2 that the relative error is about $10^{-12 \%}$ (near the machine precision). This accuracy is due to the fact that the linear elements are sufficient to perfectly describe the exact solution (as the piecewise constant Lagrange multiplier representing the contact pressure) and to the fact that all intersections on the contact zone are precisely computed. Then the integration scheme used to compute the contact contributions is accurate enough for linear functions. So we observe (as predicted by the theory) that the LAC method behaves as well as the standard mortar approach. We recall that the method using node-to-face (or node-to-segment in 2D) exhibits in this test with nonmatching meshes some oscillations for the contact pressure (see, e.g. [14]). 


\begin{tabular}{|l|c|c|}
\hline Element type & $\begin{array}{c}\text { Maximum relative } \\
\text { error }\left|\lambda-\lambda^{h}\right|\end{array}$ & $\begin{array}{c}\text { Maximum relative } \\
\text { error }\left|\sigma_{y y}-\sigma_{y y}^{h}\right|\end{array}$ \\
\hline 3-node triangle & $5 \cdot 10^{-12} \%$ & $2 \cdot 10^{-12 \%}$ \\
\hline 4-node quadrangle & $4 \cdot 10^{-12} \%$ & $4 \cdot 10^{-12 \%}$ \\
\hline
\end{tabular}

Table 1: Relative error on $\lambda$ and $\sigma_{y y}$ in $2 \mathrm{D}$.

\begin{tabular}{|l|c|c|}
\hline Element type & $\begin{array}{c}\text { Maximum relative } \\
\text { error }\left|\lambda-\lambda^{h}\right|\end{array}$ & $\begin{array}{c}\text { Maximum relative } \\
\text { error }\left|\sigma_{z z}-\sigma_{z z}^{h}\right|\end{array}$ \\
\hline 4-node tetrahedron & $8 \cdot 10^{-12} \%$ & $2 \cdot 10^{-12 \%}$ \\
\hline 8-node hexahedron & $6 \cdot 10^{-12} \%$ & $4 \cdot 10^{-12 \%}$ \\
\hline
\end{tabular}

Table 2: Relative error on $\lambda$ and $\sigma_{z z}$ in 3D.

Remark 4. In this example we only considered linear finite elements. Note that the quadratic finite elements also successfully pass the Taylor patch test. The quadratic finite element using LAC contact condition will be investigated in the next test case which deals with curved contact areas.

\subsection{Self-included spheres, self-included disks}

In this example, we consider two self-included disks/spheres in 2D/3D denoted $\Omega^{1}$ (internal body) and $\Omega^{2}$ (external body). The internal/external radii of $\Omega^{1}$ are $\left(r_{\text {int }}^{1}, r_{\text {ext }}^{1}\right)=(20 \mathrm{~mm}, 30 \mathrm{~mm})$ and the internal/external radii of $\Omega^{2}$ are $\left(r_{i n t}^{2}, r_{e x t}^{2}\right)=(30 \mathrm{~mm}, 40 \mathrm{~mm})$. So the contact area is a sphere of radius $30 \mathrm{~mm}$. Due to the curved geometries we only use quadratic finite elements. The material characteristics for both bodies are a Young modulus $E=2000 M P a$ and a Poisson ratio $\nu$ of 0.3 . The internal boundary $r=r_{i n t}^{1}$ is subject to a pressure load $p=25 \mathrm{MPa}$ while the external boundary $r=r_{\text {ext }}^{2}$ is a free surface. In 2D, the analytical results are as follows for $20 \leq r \leq 40$ and $0 \leq \theta \leq 2 \pi$ (polar coordinates):

$$
\begin{aligned}
u_{r}(r, \theta) & =\frac{\left(r_{\text {int }}^{1}\right)^{3}}{\left(r_{\text {ext }}^{2}\right)^{3}-\left(r_{\text {int }}^{1}\right)^{3}}\left((1-2 \nu) r+(1+\nu) \frac{\left(r_{\text {ext }}^{1}\right)^{3}}{2 r^{2}}\right) \frac{p}{E}, \\
\sigma_{r r}(r, \theta) & =-\frac{\left(r_{\text {int }}^{1}\right)^{3}}{\left(r_{\text {ext }}^{2}\right)^{3}-\left(r_{\text {int }}^{1}\right)^{3}}\left(\frac{\left(r_{\text {ext }}^{2}\right)^{3}}{r^{3}}-1\right) p .
\end{aligned}
$$


Therefore

$$
\begin{aligned}
u_{r}(20, \theta) & =0.2 \mathrm{~mm}, \\
u_{r}(40, \theta) & =0.075 \mathrm{~mm}, \\
\sigma_{r r}(20, \theta) & =-25 M P a, \\
\lambda=\sigma_{r r}(30, \theta) & =-4,894179894 M P a, \\
\sigma_{r r}(40, \theta) & =0 M P a .
\end{aligned}
$$
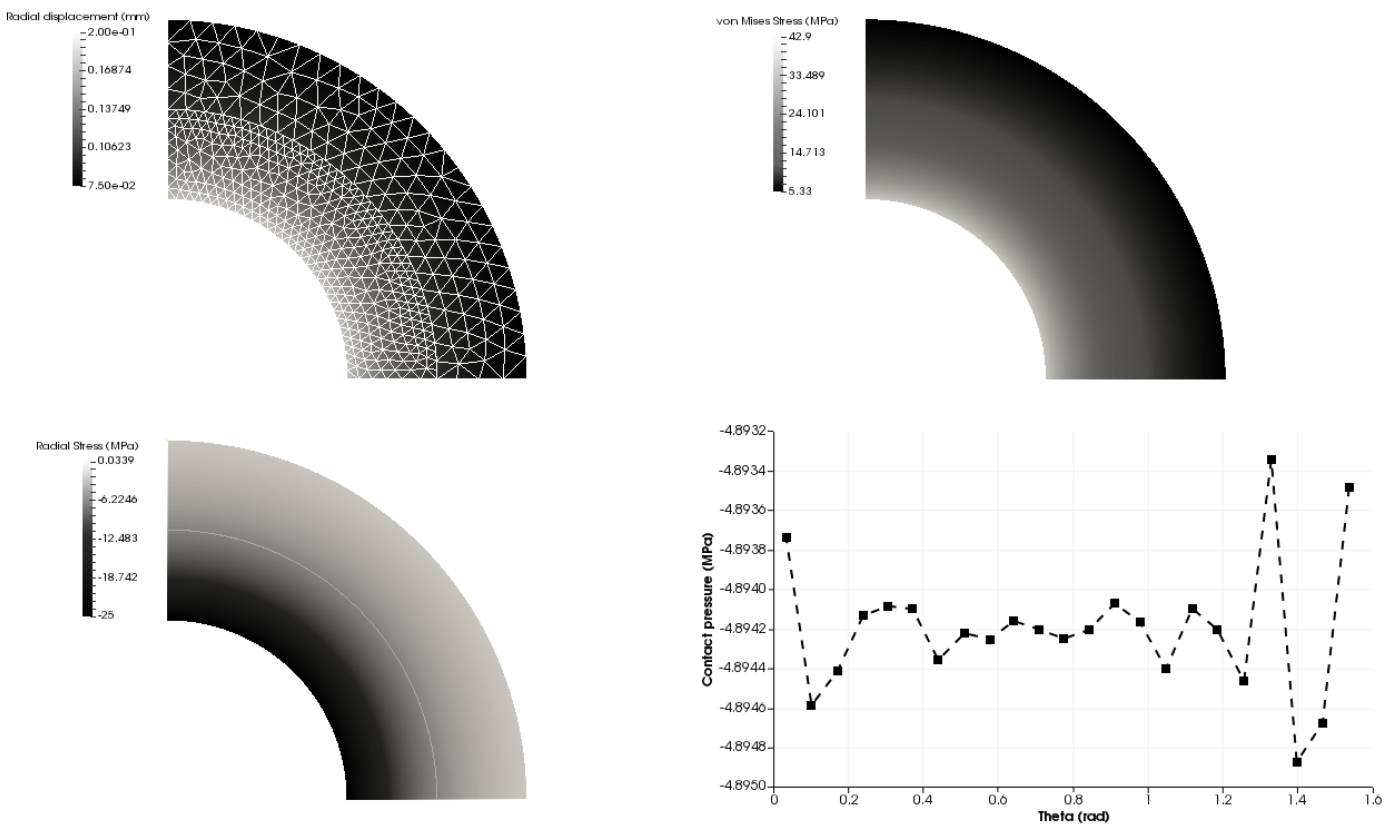

Figure 5: Numerical results obtained with LAC in 2D when considering 6-node triangles. Top left : radial displacement; top right : Von Mises stresses; bottom left : radial stresses; bottom right : contact pressure. 

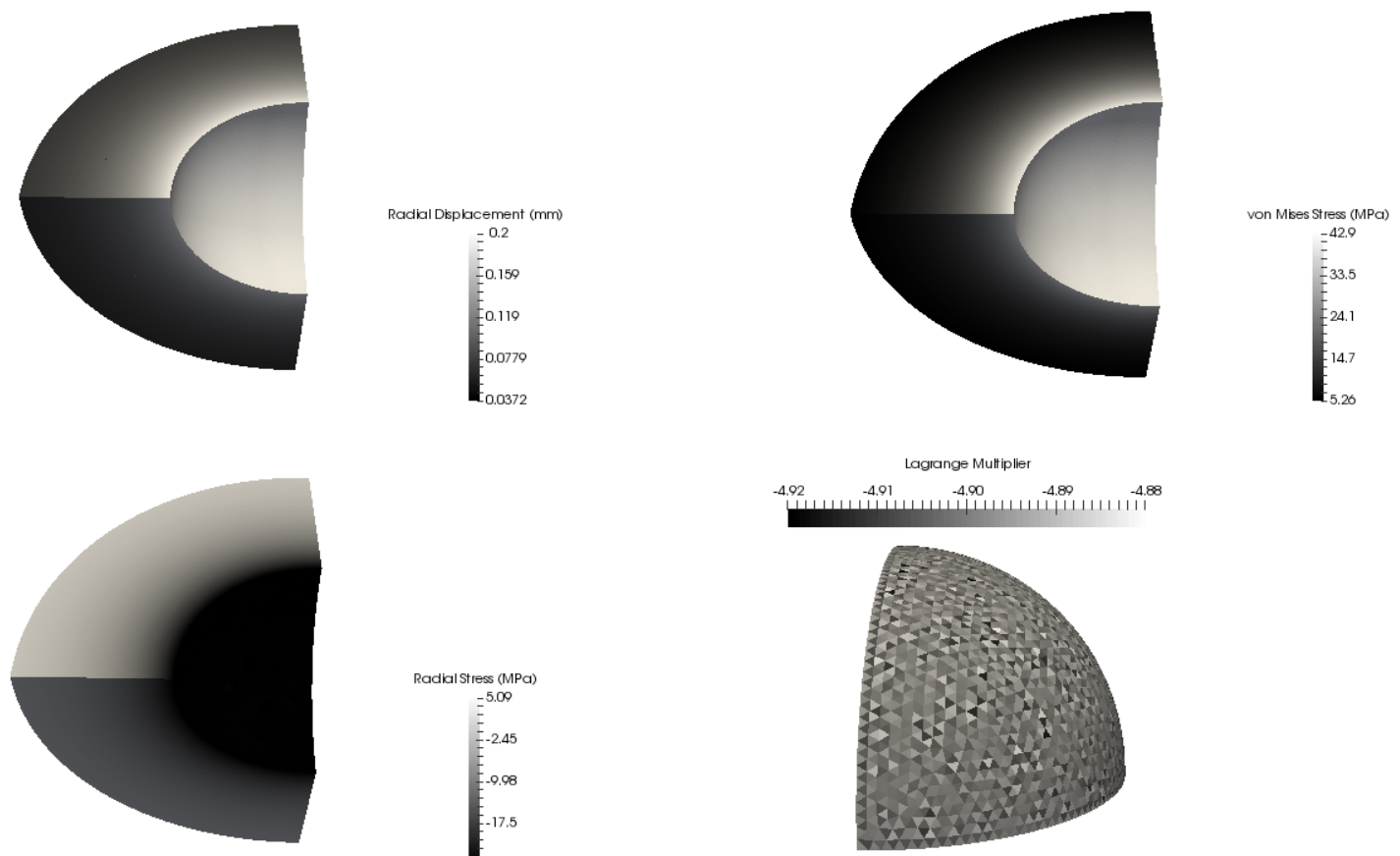

Figure 6: Numerical results obtained with LAC in 3D when considering 10-node tetrahedra. Top left : radial displacement; top right : Von Mises stresses; bottom left : radial stresses; bottom right : contact pressure

The numerical results obtained with the LAC for quadratic meshes using 6-node triangles with 2D axisymmetric modeling and 10-node tetrahedra in 3D are depicted in Figures 5 and 6. Although there are nonmatching meshes on the contact areas we observe no spurious oscillations near the contact area neither for the displacement nor for the constraints and we obtain a contact pressure (Lagrange multiplier) which is almost constant on the contact area and close to the exact value. The relative errors are very low as shown in Tables 3 and 4 . 


\begin{tabular}{|l|c|c|c|}
\hline $\begin{array}{l}\text { Element } \\
\text { type }\end{array}$ & $\begin{array}{c}\text { Maximum relative } \\
\text { error }\left|\lambda-\lambda^{h}\right|\end{array}$ & $\begin{array}{c}\text { Maximum relative } \\
\text { error }\left|\sigma_{r r}-\sigma_{r r}^{h}\right| \\
\text { Slave }\end{array}$ & $\begin{array}{c}\text { Maximum relative } \\
\text { error }\left|\sigma_{r r}-\sigma_{r r}^{h}\right| \\
\text { Master }\end{array}$ \\
\hline $\begin{array}{l}\text { 6-node } \\
\text { triangle }\end{array}$ & $0.02 \%$ & $1.52 \%$ & $0.43 \%$ \\
\hline $\begin{array}{l}\text { 8-node } \\
\text { quadrangle }\end{array}$ & $1.82 \cdot 10^{-4} \%$ & $0.19 \%$ & $0.37 \%$ \\
\hline
\end{tabular}

Table 3: Relative error on $\lambda$ and $\sigma_{r r}$ in the 2D case.

\begin{tabular}{|l|c|c|c|}
\hline $\begin{array}{l}\text { Element } \\
\text { type }\end{array}$ & $\begin{array}{c}\text { Maximum relative } \\
\text { error }\left|\lambda-\lambda^{h}\right|\end{array}$ & $\begin{array}{c}\text { Maximum relative } \\
\text { error }\left|\sigma_{r r}-\sigma_{r r}^{h}\right| \\
\text { Slave }\end{array}$ & $\begin{array}{c}\text { Maximum relative } \\
\text { error }\left|\sigma_{r r}-\sigma_{r r}^{h}\right| \\
\text { Master }\end{array}$ \\
\hline $\begin{array}{l}10 \text {-node } \\
\text { tetrahedron }\end{array}$ & $0.5 \%$ & $1.37 \%$ & $0.76 \%$ \\
\hline $\begin{array}{l}20 \text {-node } \\
\text { hexahedron }\end{array}$ & $1.61 \%$ & $3.57 \%$ & $2.33 \%$ \\
\hline $\begin{array}{l}27 \text {-node } \\
\text { hexahedron }\end{array}$ & $1.46 \%$ & $2.4 \%$ & $2.58 \%$ \\
\hline
\end{tabular}

Table 4: Relative error on $\lambda$ and $\sigma_{r r}$ in the 3D case.

Remark 5. Since we did not use any strategy to take into account the geometrical error in the linear finite element case, the results for this test with linear elements are not representative unless we use very fine and almost compatible meshes.

\subsection{Numerical convergence rate}

As third example we study the numerical convergence rates of the LAC method which are compared with the theoretical ones. In order to perform this study, we choose the manufactured solutions approach in both the 2D and the $3 \mathrm{D}$ cases, see [50]. In $2 \mathrm{D}$ we consider a square $\Omega=(0,1)^{2},(E=$ $1, \nu=0$ ) having initially one contact point with a curved rigid foundation of equation $y \leq-0.05(x-0.5)^{2}$ and which is pressed against the foundation. Figure 7 shows the displacement of the deformed bodies. We analytically 
define the load and boundary conditions to be applied to the structure in order to get the following solution:

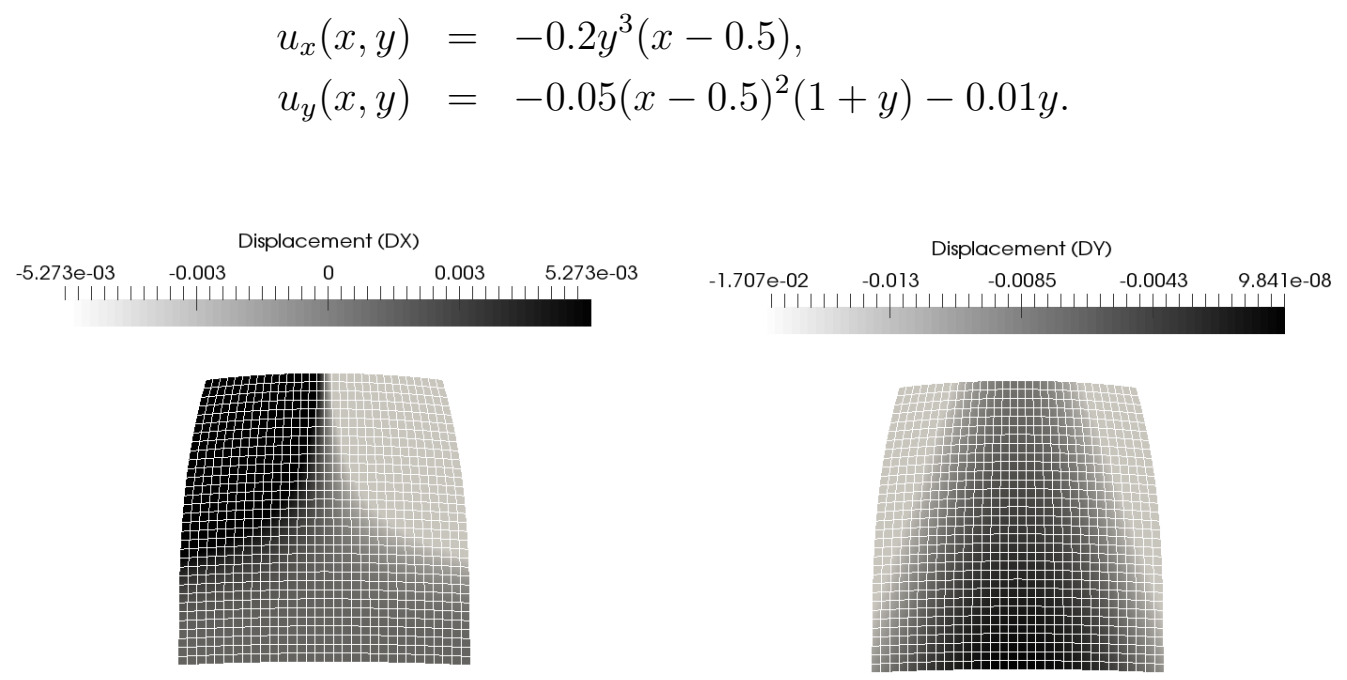

(a) Horizontal

(b) Vertical

Figure 7: Displacement for the manufactured solution in 2D

The 3D case is similar: a cube $\Omega=(0,1)^{3}$ initially in contact with the curved foundation $z \leq-0.2\left(1+x^{2}+y^{2}\right)-0.3$. Figure 8 shows the displacement of the deformed bodies. We analytically define the load and boundary conditions to be applied to the structure in order to get the following solution:

$$
\begin{aligned}
& u_{x}(x, y, z)=0.2 x y z^{2} \\
& u_{y}(x, y, z)=0.2 x y z^{2} \\
& u_{z}(x, y, z)=-0.2\left(1+x^{2}+y^{2}\right)(1+0.01 z)-0.01 z-0.3 .
\end{aligned}
$$

Then we compute the $L^{2}$-error in displacement $\left\|u-u^{h}\right\|_{0, \Omega}$ and the $L^{2}$ error on the contact pressure $\left\|\lambda-\lambda^{h}\right\|_{0, \Gamma_{C}}$ (seen as a Lagrange multiplier). Although there is to our knowledge no proof of optimal $L^{2}(\Omega)$-error decay on the displacements (the only partial existing results can be found in $[51,52]$ ) we can nevertheless expect that this error behaves like $h\left\|u-u^{h}\right\|_{1, \Omega}$ as in the linear case where the Aubin-Nitsche argument can be applied. So we compare our numerical convergence rates with this unproved and expected optimal theoretical rate. Concerning the $L^{2}\left(\Gamma_{C}\right)$-error on the Lagrange multiplier 


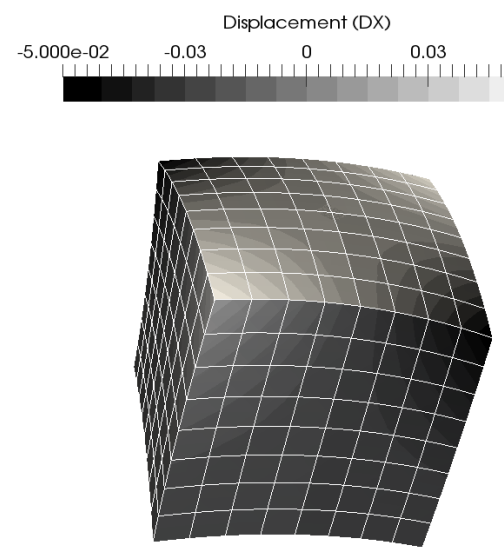

(a) Horizontal-x
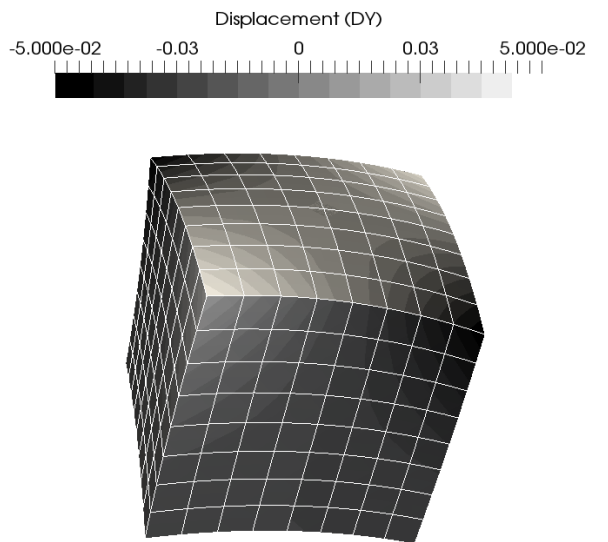

(b) Horizontal-y
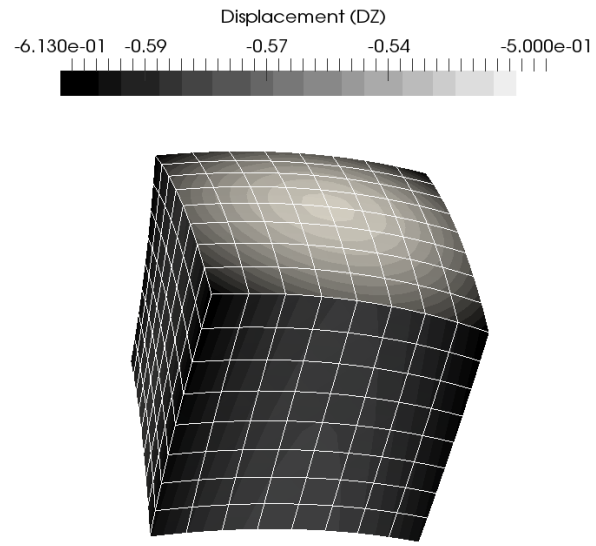

(c) Vertical

Figure 8: Displacement for 3D manufactured solution

the situation is simpler. By using standard results (inverse inequality and approximation properties as in $[51,52])$ we easily obtain from Theorem 1:

$$
\left\|\lambda-\lambda^{h}\right\|_{0, \Gamma_{C}} \leq C h^{\tau-3 / 2}\|u\|_{\tau, \Omega},
$$

where $3 / 2<\tau \leq \min (k+1,5 / 2)$. 


\begin{tabular}{|c|c|c|c|c|}
\hline \multirow{2}{*}{ Element type } & \multicolumn{2}{|c|}{$\left\|u-u^{h}\right\|_{0, \Omega}$} & \multicolumn{2}{c|}{$\left\|\lambda-\lambda^{h}\right\|_{0, \Gamma_{C}}$} \\
\cline { 2 - 5 } & Expected & Numerical & Expected & Numerical \\
\hline 3-node triangle & 2.0 & 1.99 & $0.5(1)^{1}$ & 1.01 \\
6-node triangle & 2.5 & 2.99 & 1.0 & 1.04 \\
\hline 4-node quadrangle & 2.0 & 2.00 & $0.5(1)$ & 1.01 \\
8-node quadrangle & 2.5 & 2.96 & 1.0 & 1.08 \\
\hline
\end{tabular}

Table 5: Convergence rates in the 2D case

\begin{tabular}{|c|c|c|c|c|}
\hline \multirow{2}{*}{ Element type } & \multicolumn{2}{|c|}{$\left\|u-u^{h}\right\|_{0, \Omega}$} & \multicolumn{2}{c|}{$\left\|\lambda-\lambda^{h}\right\|_{0, \Gamma_{C}}$} \\
\cline { 2 - 5 } & Expected & Numerical & Expected & Numerical \\
\hline 4-node tetrahedron & 2.0 & 1.99 & $0.5(1)$ & 1.51 \\
10-node tetrahedron & 2.5 & 2.99 & 1.0 & 1.99 \\
\hline 8-node hexahedron & 2.0 & 1.98 & $0.5(1)$ & 1.93 \\
20-node hexahedron & 2.5 & 2.54 & 1.0 & 1.04 \\
27-node hexahedron & 2.5 & 2.97 & 1.0 & 1.01 \\
\hline
\end{tabular}

Table 6: Convergence rates in the 3D case

As shown in tables 5 and 6 , the numerical convergence rates are either very close or better than the expected ones; in the latter case we observe some superconvergence. The superconvergence on the displacement error could be explained by the fact that the solution of the continuous problem is indefinitely continuously differentiable and therefore more regular than $H^{5 / 2}$. Concerning the superconvergence which is sometimes observed for the Lagrange multiplier, it may be possible that we observe at first the geometric error convergence and not the asymptotic error decay rate.

\subsection{Ring on block}

In this last numerical experiment, we consider an hyperelastic half-ring (external diameter $190 \mathrm{~mm}$ and internal diameter $170 \mathrm{~mm}$ ) made of two materials of same thickness $(5 \mathrm{~mm})$ and a hyperelastic base of $250 \mathrm{~mm}$ length, $50 \mathrm{~mm}$ height and $50 \mathrm{~mm}$ depth, see Figure 9 . We apply a $-90 \mathrm{~mm}$ vertical

\footnotetext{
${ }^{1}$ In the paper [52] the authors prove for a standard conforming linear finite element approximation of the Signorini problem in 2D and 3D that the $L^{2}\left(\Gamma_{C}\right)$-error bound on the multipliers could be improved with a factor $h^{1 / 2}$. This could also explain the better convergence we observe in our framework which is very close to that considered in [52].
} 
displacement at both ends of the half-ring. There is an initial gap of $20 \mathrm{~mm}$ between the bottom of the half-ring and the base. In order to reduce the model size, we use a symmetry condition and we only consider a quarter of the structure. We will compute the solution on six different meshes (linear and quadratic hexahedra with two levels of refinement), the mesh characteristics are described in Table 7.

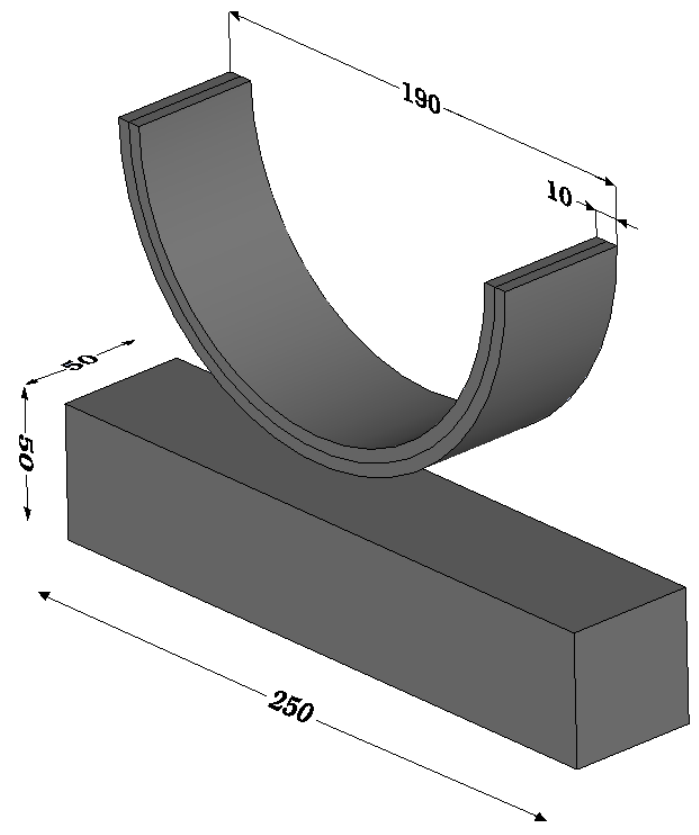

Figure 9: Test case configuration 


\begin{tabular}{|l|c|c|}
\hline Mesh type & Nodes & Elements \\
\hline 8-node hexahedron coarse & 3085 & 4162 \\
20-node hexahedron coarse & 11133 & 4162 \\
27-node hexahedron coarse & 13998 & 3037 \\
\hline 8-node hexahedron fine & 17998 & 21784 \\
20-node hexahedron fine & 67454 & 21784 \\
27-node hexahedron fine & 102114 & 17284 \\
\hline
\end{tabular}

Table 7: Considered meshes

We are mainly studying two variables of interest: the displacement at the middle of the half-ring as a function of the load step (we use 60 load steps), so each load step represents a vertical displacement of approximatively $1.16 \mathrm{~mm}$ and the contact pressure. The results, displacement field on the deformed shape and contact pressure (in the case of 20-node hexahedra) are depicted in Figure 10.

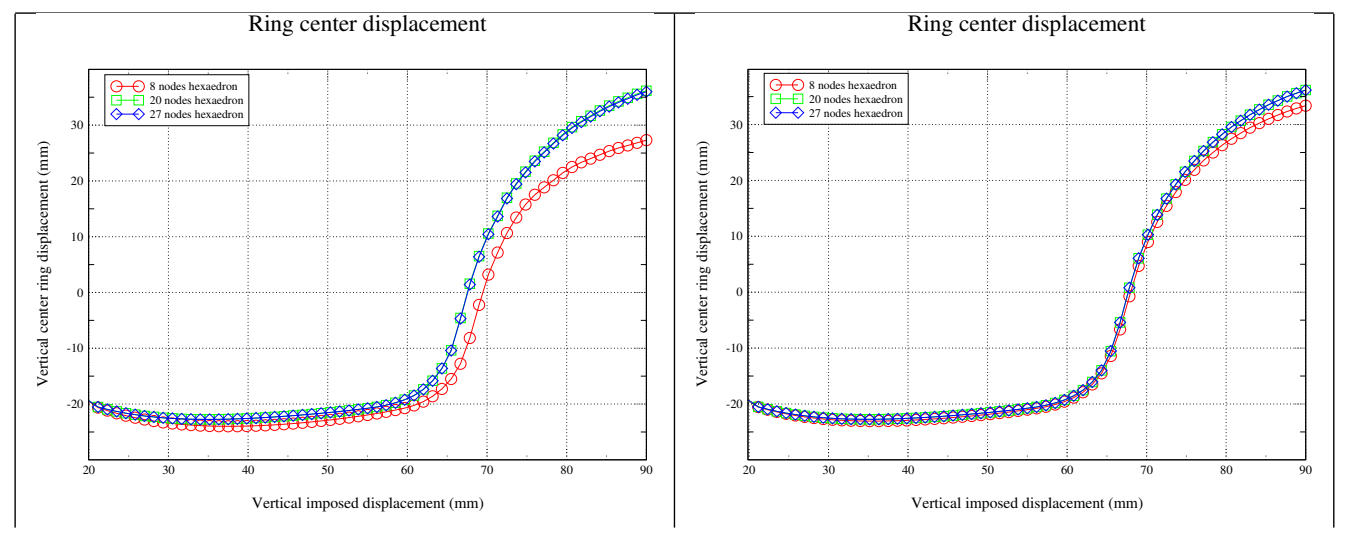

Figure 10: Displacement of the half-ring center depending on the imposed displacement (left: coarse meshes, right: fine meshes) 


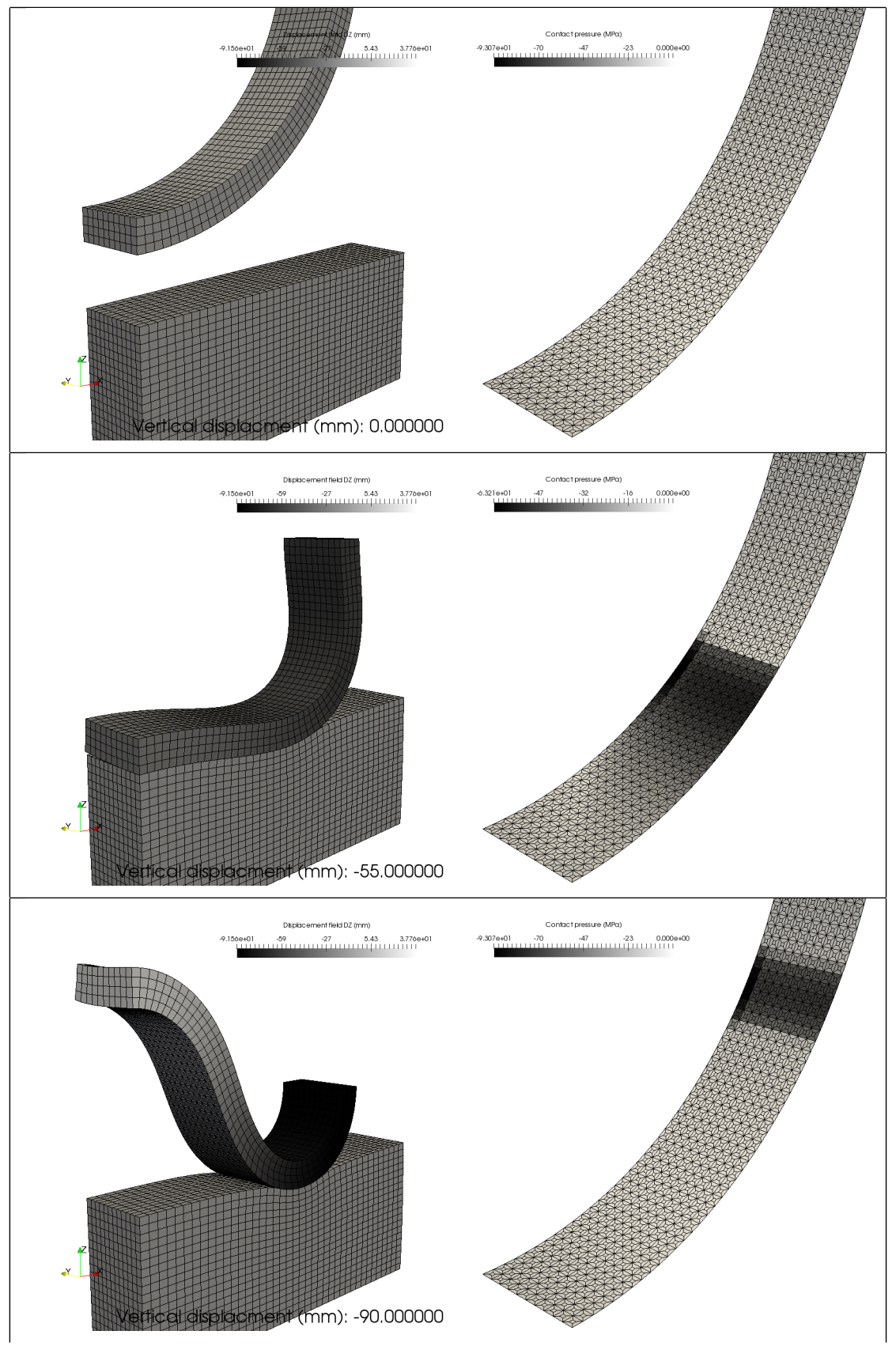

Figure 11: Displacement field and contact pressure for 20-node hexahedra at different load steps.

The obtained results are very close to those in [49]: we get the same pattern 
and the same maximum value for the displacement of the center of the halfring. We also notice that the contact pressure shows no perceptible oscillation when using quadratic meshes (some oscillation may occur when using coarse linear meshes due to the geometric error disturbing the contact detection and computation). These results confirm the capacity of the LAC method to deal with more complicated contact problems.

\section{Conclusion}

The Local Average Contact approach allows to handle the nonmatching meshes independently of the space dimension and of the degree and type of finite elements. Basically, it consists of averaging the interpenetration of the bodies on small areas (macro-elements) or equivalently to consider Lagrange multipliers which are constant on these small areas. We show that this appoach gives interesting and accurate results as proved by the theory. This method could be promising in particular for complex three dimensional contact problems with quadratic elements since it is simple and local and it benefits from mathematical proofs of convergence. The next step would be to consider real-life engineering simulations.

\section{Appendix A. A stable average preserving operator for the inf-sup condition}

We need to define an operator denoted $\pi_{1}^{h}$ when $d=2,3$ and $k=1$ which is the generalization of the operator in [29] to the geometrical nonconforming case (i.e., $\Gamma_{C}^{\varepsilon} \neq \emptyset$ ) in order to study the behavior of the inf-sup constant $\beta^{h}$. Actually we are not able to achieve the analysis in the geometrical nonconforming case with quadratic elements for technical reasons. For the analysis in the geometrical conforming case (with nonmatching meshes) and quadratic elements we refer the reader to $[33,29]$. We next suppose that $T^{M}$ is a macro-mesh of $\Gamma_{C}$ satisfying Hypothesis 1 and built from the mesh of $\mathcal{T}_{1}^{h} \cap \Gamma_{C}$. Let $W_{\ell}^{h}$ be the normal trace space of $V_{\ell}^{h}$ on $\Gamma_{C}^{\ell}$. Let $\phi_{i}$ stand for the basis functions associated to the degrees of freedom of $W_{1}^{h}$. We denote $x_{i}$, $i=1, . ., n$ the corresponding nodes of $\mathcal{T}_{1}^{h} \cap \Gamma_{C}^{1}$ and $\omega_{i}$ is the union of (closed) macro-meshes containing $x_{i}$. The next definition defines the operator on admissible macro-elements.

Definition 2. Assume that Hypothesis 1 holds. The operator

$$
\pi_{1}^{h}: L^{1}\left(\Gamma_{C}\right) \longrightarrow W_{1}^{h}
$$


is as follows for any $v \in L^{1}\left(\Gamma_{C}\right)$. If $x_{i}$ is a node in $\overline{\Gamma_{C}^{1}} \cap \overline{\Gamma_{D}^{1}}$, then $\pi_{1}^{h} v\left(x_{i}\right)=$ 0 . The operator $\pi_{1}^{h} v$ is defined locally on every admissible macro-element $T^{m} \in T_{a d}^{M}$ having as nodes $x_{i}, i=1, \ldots, m^{\star}\left(x_{i} \notin \overline{\Gamma_{C}^{1}} \cap \overline{\Gamma_{D}^{1}}\right)$ and as internal d.o.f. $x_{m^{\star}+1}$ by

$$
\pi_{1}^{h} v=\sum_{j=1}^{m^{\star}+1} \alpha_{j}(v) \phi_{j}
$$

where

$$
\left\{\begin{array}{c}
\alpha_{i}(v)=\frac{\int_{\omega_{i} \cap \Gamma_{C}} v d \Gamma}{\left|\omega_{i} \cap \Gamma_{C}\right|}, \quad 1 \leq i \leq m^{\star}, \\
\alpha_{m^{\star}+1}(v)=\frac{\int_{T^{m} \cap \Gamma_{C}} v d \Gamma-\sum_{j=1}^{m^{\star}} \int_{T^{m} \cap \Gamma_{C}} \alpha_{j}(v) \phi_{j} d \Gamma}{\int_{T^{m} \cap \Gamma_{C}} \phi_{m^{\star}+1} d \Gamma} .
\end{array}\right.
$$

We have:

Proposition 2. 1. The operator $\pi_{1}^{h}$ is linear and satisfies

$$
\int_{T^{m} \cap \Gamma_{C}} \pi_{1}^{h} v-v d \Gamma=0, \forall v \in L^{1}\left(\Gamma_{C}\right), \forall T^{m} \in T_{a d}^{M} .
$$

2. The operator $\pi_{1}^{h}$ is locally $H^{s}$-stable, for $0 \leq s \leq 1$, i.e., there exists $C>0$ such that for any $v \in H^{s}\left(T^{m}\right), \forall T^{m} \in T_{a d}^{M}$,

$$
\left\|\pi_{1}^{h} v\right\|_{s, T^{m}} \leq C \varepsilon^{-1}\|v\|_{s, \tilde{T}^{m}}
$$

where $\tilde{T}^{m}$ is the patch surrounding $T^{m}$ in $\Gamma_{C}: \tilde{T}^{m}=\bigcup_{i: x_{i} \in T^{m}}\left(\omega_{i} \cap \Gamma_{C}\right)$.

Proof of the proposition. The linearity of $\pi_{1}^{h}$ is obvious. The average preserving property on $T^{m} \cap \Gamma_{C}$ for any admissible $T^{m}$ follows directly from the definition of $\pi_{1}^{h}$.

Let $v \in L^{2}\left(\Gamma_{C}\right), T^{m} \in T_{a d}^{M}$ and let $\tilde{T}^{m}$ be the patch surrounding $T^{m}$ in $\Gamma_{C}$ : 
$\tilde{T}^{m}=\bigcup_{i: x_{i} \in T^{m}}\left(\omega_{i} \cap \Gamma_{C}\right)$. Let $x_{i} \in T^{m}, 1 \leq i \leq m^{\star}$, we have

$$
\begin{aligned}
\left|\alpha_{i}(v)\right| \leq\left|\omega_{i} \cap \Gamma_{C}\right|^{-1} \int_{\omega_{i} \cap \Gamma_{C}}|v| d \Gamma & \leq\left|\omega_{i} \cap \Gamma_{C}\right|^{-\frac{1}{2}}\|v\|_{0, \omega_{i} \cap \Gamma_{C}} \\
& \leq\left|T^{m} \cap \Gamma_{C}\right|^{-\frac{1}{2}}\|v\|_{0, \tilde{T}^{m}} \\
& \leq \varepsilon^{-1} \overline{\phi_{m+1}}\left|T^{m}\right|^{-\frac{1}{2}}\|v\|_{0, \tilde{T}^{m}} \\
& \leq \varepsilon^{-1}\left|T^{m}\right|^{-\frac{1}{2}}\|v\|_{0, \tilde{T}^{m}} .
\end{aligned}
$$

Then

$$
\begin{aligned}
& \left|\alpha_{m^{\star}+1}(v)\right| \\
& =\left|\int_{T^{m} \cap \Gamma_{C}} v d \Gamma-\sum_{k \neq m^{\star}+1: x_{k} \in T^{m}} \int_{T^{m} \cap \Gamma_{C}} \alpha_{k}(v) \phi_{k} d \Gamma\right|\left|\int_{T^{m} \cap \Gamma_{C}} \phi_{m^{\star}+1} d \Gamma\right|^{-1} \\
& \leq\left(\left|T^{m} \cap \Gamma_{C}\right|^{\frac{1}{2}}\|v\|_{0, T^{m} \cap \Gamma_{C}}+\sum_{k \neq m^{\star}+1: x_{k} \in T^{m}}\left|T^{m} \cap \Gamma_{C}\right|^{\frac{1}{2}}\|v\|_{0, \tilde{T}^{m}}\right)\left|\int_{T^{m} \cap \Gamma_{C}} \phi_{m^{\star}+1} d \Gamma\right|^{-1} \\
& \leq C\left(\overline{\phi_{m^{\star}+1}}\right)^{-1}\left|T^{m} \cap \Gamma_{C}\right|^{-1 / 2}\|v\|_{0, \tilde{T}^{m}} \\
& \leq C \varepsilon^{-1}\left|T^{m}\right|^{-1 / 2}\|v\|_{0, \tilde{T}^{m}}
\end{aligned}
$$

where we use (A.1) together with $\left|\phi_{i}\right| \leq 1$ on $\Gamma_{C}$ and Cauchy-Schwarz inequality. Next, we prove the local $L^{2}$-stability of $\pi_{1}^{h}$.

$$
\begin{aligned}
\left\|\pi_{1}^{h} v\right\|_{0, T^{m}}=\left\|\sum_{i: x_{i} \in T^{m}} \alpha_{i}(v) \phi_{i}\right\|_{0, T^{m}} & \leq \sum_{i: x_{i} \in T^{m}}\left|\alpha_{i}(v)\right|\left\|\phi_{i}\right\|_{0, T^{m}} \\
& \leq\left|T^{m}\right|^{\frac{1}{2}} \sum_{i: x_{i} \in T^{m}}\left|\alpha_{i}(v)\right| \\
& \leq C \varepsilon^{-1}\|v\|_{0, \tilde{T}^{m}} .
\end{aligned}
$$

We now need to prove the local $H^{1}$-stability of $\pi_{1}^{h}$. We assume that $v \in$ $H^{1}\left(\Gamma_{C}\right)$. Let $T^{m} \in T_{a d}^{M}$ and let $a \in P_{0}\left(\tilde{T}^{m}\right)$. Since $\pi_{1}^{h} a=a$ on $T^{m}$, we get from an inverse estimate and the local $L^{2}\left(T^{m}\right)$-stability of $\pi_{1}^{h}$ (the notations correspond to the case $d=2$ but it can be straightforwardly extended to the three-dimensional case, see [29] and the references therein).

$$
\begin{aligned}
\left\|\left(\pi_{1}^{h} v\right)^{\prime}\right\|_{0, T^{m}}=\left\|\left(\pi_{1}^{h}(v-a)\right)^{\prime}\right\|_{0, T^{m}} & \leq C h_{T^{m}}^{-1}\left\|\pi_{1}^{h}(v-a)\right\|_{0, T^{m}} \\
& \leq C h_{T^{m}}^{-1} \varepsilon^{-1}\|v-a\|_{0, \tilde{T}^{m}} .
\end{aligned}
$$


If $a$ is the average of $v$ on $\tilde{T}^{m}$, we get $\|v-a\|_{0, \tilde{T}^{m}} \leq C h_{\tilde{T}^{m}}\left\|v^{\prime}\right\|_{0, \tilde{T}^{m}}$ from which

$$
\left\|\left(\pi_{1}^{h} v\right)^{\prime}\right\|_{0, T^{m}} \leq C \varepsilon^{-1}\left\|v^{\prime}\right\|_{0, \tilde{T}^{m}},
$$

follows and consequently the local $H^{1}$-stability. An hilbertian interpolation argument (see $[53,54]$ ) allows us to prove the $H^{s}\left(\Gamma_{C}\right)$-stability of $\pi_{1}^{h}$ for all $s \in(0,1)$.

In order to satisfy the infsup condition (10) we need to to show (see $[33,29])$ that there is a constant $C$ such that: for all $\mu^{h} \in X^{h}$ and all $v \in V$ there exists $v^{h} \in V^{h}$ such that

$$
\begin{aligned}
b\left(\mu^{h}, v^{h}\right) & =b\left(\mu^{h}, v\right), \\
\left\|v^{h}\right\| & \leq C\|v\| .
\end{aligned}
$$

In fact if (A.3) and (A.4) hold, we get $\beta^{h}=\tilde{\beta} / C$ where $\tilde{\beta}$ is the inf-sup constant of the continuous problem (see [1]).

We set $v^{h}=\left(v_{1}^{h}, v_{2}^{h}\right)$ such that

$$
v_{1}^{h}=R_{1}^{h} \pi_{1}^{h}\left[v_{N}\right], \quad v_{2}^{h}=0,
$$

where $R_{1}^{h}$ ia a discrete extension operator from $H^{1 / 2}\left(T_{a d}^{M}\right)$ in $V_{1}^{h}$. Since $\pi_{1}^{h}$ preserves the average on every admissible macro-element intersected with $\Gamma_{C}$ and the multipliers $\mu^{h} \in X^{h}$ vanish on $\Gamma_{C}^{\varepsilon}$, we get (A.3). It remains to show that $v^{h}$ verifies (A.4). Thanks to the $H^{\frac{1}{2}}\left(T_{a d}^{M}\right)$-stability of $\pi_{1}^{h}$ with a constant $C \varepsilon^{-1}$ and the trace theorem, we have

$\left\|v^{h}\right\|=\left\|R_{1}^{h} \pi_{1}^{h}\left[v_{N}\right]\right\|_{1, \Omega^{1}} \leq C\left\|\pi_{1}^{h}\left[v_{N}\right]\right\|_{1 / 2, T_{a d}^{M}} \leq C \varepsilon^{-1}\left\|\left[v_{N}\right]\right\|_{1 / 2, \Gamma_{C}} \leq C \varepsilon^{-1}\|v\|$.

So the constant $\beta^{h}$ in (10) can be chosen $\beta^{h}=C \varepsilon$ where $C$ is independent of $h$ and $\varepsilon$.

[1] J. Haslinger, I. Hlavácek, J. Necas, Numerical methods for unilateral problems in solid mechanics, Vol. 4 of Handbook of Numerical Analysis, North-Holland, Amsterdam, 1996.

[2] N. Kikuchi, J. T. Oden, Contact problems in elasticity: a study of variational inequalities and finite element methods, SIAM, 1988.

[3] T. A. Laursen, Computational contact and impact mechanics: fundamentals of modeling interfacial phenomena in nonlinear finite element analysis, Springer, 2002. 
[4] B. I. Wohlmuth, Variationally consistent discretization schemes and numerical algorithms for contact problems, Acta Numerica 20 (2011) 569734.

[5] P. Wriggers, Computational contact mechanics, Springer, 2006.

[6] A. Signorini, Questioni di elastostatica linearizzata e semilinearizzata, Rend. Math 18 (1959) 381-402.

[7] G. Fichera, Problemi elastostatici con vincoli unilaterali: il problema di Signorini con ambigue condizioni al contorno, Atti Accad. Naz. Lincei Mem. Cl. Sci. Fis. Mat. Natur. Sez. I 7 (8) (1963/1964) 91-140.

[8] M. Moussaoui, K. Khodja, Régularité des solutions d'un problème mêlé dirichlet-signorini dans un domaine polygonal plan, Communications in partial differential equations 17 (5-6) (1992) 805-826.

[9] C. Bernardi, Y. Maday, A. T. Patera, A new non conforming approach to domain decomposition: The mortar element method, in: J. H. Brezis (Ed.), Collège de France Seminar, Pitman, 1994, pp. 13-51.

[10] G. Bayada, M. Chambat, K. Lhalouani, T. Sassi, Éléments finis avec joints pour des problèmes de contact avec frottenment de Coulomb non local. (french) [on the mortar finite element method for contact problems with nonlocal Coulomb law], C. R. Acad. Sci. Paris Sér. I Math. 325 (12) (1997) 1323-1328.

[11] F. Ben Belgacem, P. Hild, P. Laborde, Approximation of the unilateral contact problem by the mortar finite element method, Comptes Rendus de l'Academie des Sciences Series I Mathematics 324 (1) (1997) 123-127.

[12] F. Ben Belgacem, P. Hild, P. Laborde, Extension of the mortar finite element method to a variational inequality modeling unilateral contact, Mathematical Models and Methods in Applied Sciences 9 (2) (1999) 287-303.

[13] P. Hild, Problèmes de contact unilatéral et maillages éléments finis incompatibles, Ph.D. thesis, Université Paul Sabatier, (www.math.univtoulouse.fr/ phild/) (1998). 
[14] P. Hild, Numerical implementation of two nonconforming finite element methods for unilateral contact, Computer Methods in Applied Mechanics and Engineering 184 (1) (2000) 99-123.

[15] A. Chernov, M. Maischak, E. P. Stephan, hp-mortar boundary element method for two-body contact problems with friction, Mathematical Methods in the Applied Sciences 31 (17) (2008) 2029-2054.

[16] T. Cichosz, M. Bischoff, Consistent treatment of boundaries with mortar contact formulations using dual Lagrange multipliers, Computer Methods in Applied Mechanics and Engineering 200 (9) (2011) 1317-1332.

[17] Z. Dostál, D. Horák, D. Stefanica, A scalable feti-dp algorithm with non-penetration mortar conditions on contact interface, Journal of computational and applied mathematics 231 (2) (2009) 577-591.

[18] P. Farah, A. Popp, W. A. Wall, Segment-based vs. element-based integration for mortar methods in computational contact mechanics., Comput. Mech. 55 (1) (2015) 209-228.

[19] S. Hartmann, E. Ramm, A mortar based contact formulation for nonlinear dynamics using dual Lagrange multipliers, Finite Elements in Analysis and Design 44 (5) (2008) 245-258.

[20] R. Krause, A nonsmooth multiscale method for solving frictional twobody contact problems in 2D and 3D with multigrid efficiency, SIAM Journal on Scientific Computing 31 (2) (2009) 1399-1423.

[21] T. A. Laursen, M. A. Puso, J. Sanders, Mortar contact formulations for deformable-deformable contact: past contributions and new extensions for enriched and embedded interface formulations, Computer methods in applied mechanics and engineering 205 (2012) 3-15.

[22] A. Popp, B. I. Wohlmuth, M. W. Gee, W. A. Wall, Dual quadratic mortar finite element methods for 3D finite deformation contact, SIAM Journal on Scientific Computing 34 (4) (2012) B421-B446.

[23] M. A. Puso, T. A. Laursen, A mortar segment-to-segment frictional contact method for large deformations, Computer Methods in Applied Mechanics and Engineering 193 (45-47) (2004) 4891-4913. 
[24] M. A. Puso, T. A. Laursen, J. Solberg, A segment-to-segment mortar contact method for quadratic elements and large deformations, Computer Methods in Applied Mechanics and Engineering 197 (6) (2008) $555-566$.

[25] I. Temizer, A mixed formulation of mortarbased contact with friction, Computer Methods in Applied Mechanics and Engineering 255 (2013) 183-195.

[26] I. Temizer, P. Wriggers, T. Hughes, Three-dimensional mortarbased frictional contact treatment in isogeometric analysis with NURBS., Computer Methods in Applied Mechanics and Engineering 209/212 (2012) $115-128$.

[27] M. Tur, F. J. Fuenmayor, P. Wriggers, A mortar-based frictional contact formulation for large deformations using Lagrange multipliers, Computer Methods in Applied Mechanics and Engineering 198 (37) (2009) 2860-2873.

[28] B. I. Wohlmuth, A. Popp, M. W. Gee, W. A. Wall, An abstract framework for a priori estimates for contact problems in 3D with quadratic finite elements, Computational Mechanics 49 (6) (2012) 735-747.

[29] G. Drouet, P. Hild, An accurate local average contact method for nonmatching meshes, Numerische Mathematik 136 (2) (2017) 467-502.

[30] S. C. Brenner, R. Scott, The mathematical theory of finite element methods, Vol. 15, Springer, 2008.

[31] P. G. Ciarlet, The finite element method for elliptic problems, Elsevier, 1978.

[32] A. Ern, J. L. Guermond, Theory and practice of finite elements, Springer-Verlag New York, Inc., 2004.

[33] G. Drouet, Méthode locale de type mortar pour le cas de maillages incompatibles de degré élevé, Ph.D. thesis, Université Paul Sabatier, (www.thesesups.ups-tlse.fr/2897/1/2015TOU30142.pdf) (2015). 
[34] G. Drouet, P. Hild, Optimal convergence for discrete variational inequalities modelling Signorini contact in 2D and 3D without additional assumptions on the unknown contact set, SIAM Journal on Numerical Analysis 53 (3) (2015) 1488-1507.

[35] M. Fabre, J. Pousin, Y. Renard, A fictitious domain method for frictionless contact problems in elasticity using nitsche's method, SMAI J. of Comput. Math. 2 (2016) 19-50.

[36] S. Amdouni, P. Hild, V. Lleras, M. Moakher, Y. Renard, A stabilized lagrange multiplier method for the enriched finite-element approximation of contact problems of cracked elastic bodies, ESAIM Math. Model. Numer. Anal. 46 (2012) 813-839.

[37] R. A. Adams, Sobolev spaces, Academic Press, New York, 1975.

[38] F. Ben Belgacem, Numerical simulation of some variational inequalities arisen from unilateral contact problems by the finite element methods, SIAM Journal on Numerical Analysis 37 (4) (2000) 1198-1216.

[39] EDF, Finite element software code_aster, analysis of structures and thermomechanics for studies and research, Open source (GPL licensing) on www.code-aster.org (1989-2017).

[40] P. Hauret, P. Le Tallec, A stabilized discontinuous mortar formulation for elastostatics and elastodynamics problems, Part 2: discontinuous Lagrange multipliers, Tech. rep., CMAP (2004).

[41] P. Hauret, P. Le Tallec, A discontinuous stabilized mortar method for general 3D elastic problems, Computer Methods in Applied Mechanics and Engineering 196 (49) (2007) 4881-4900.

[42] S. Hüeber, B. I. Wohlmuth, An optimal a priori error estimate for nonlinear multibody contact problems, SIAM Journal on Numerical Analysis 43 (1) (2005) 156-173.

[43] A. Popp, M. Gitterle, M. W. Gee, W. A. Wall, A dual mortar approach for 3d finite deformation contact with consistent linearization, Int. J. Numer. Meth. Engrg 83 (2010) 1428-1465. 
[44] A. Curnier, P. Alart, A generalized newton method for friction contact problems with friction, J. Math. Pures Appl. 1 (1988) 67-82.

[45] S. Hüeber, B. I. Wohlmuth, A primal-dual active set strategy for nonlinear multibody contact problems., Comput. Methods Appl. Mech. Engrg. 194 (27-29) (2005) 3147-3166.

[46] M. J. Gander, C. Japhet, Algorithm 932: Pang: software for nonmatching grid projections in $2 \mathrm{~d}$ and $3 \mathrm{~d}$ with linear complexity, ACM Transactions on Mathematical Software (TOMS) 40 (1) (2013) 6.

[47] R. L. Taylor, P. Papadopoulos, On a patch test for contact problems in two dimensions, Computational methods in nonlinear mechanics (1991) 690-702.

[48] K. Fischer, P. Wriggers, Frictionless 2d contact formulations for finite deformations based on the mortar method, Computational Mechanics 36 (3) (2005) 226-244.

[49] K. Poulios, Y. Renard, An unconstrained integral approximation of large sliding frictional contact between deformable solids, Computers \& Structures 153 (2015) 75-90.

[50] E. Chamberlan, A. Fortin, N. Tardieu, Solutions analytiques de problèmes de contact en grandes déformations, Mécanique \& Industries $11(3-4)$ (2010) 271-276.

[51] P. Coorevits, P. Hild, K. Lhalouani, T. Sassi, Mixed finite element methods for unilateral problems: convergence analysis and numerical studies, Mathematics of Computation 71 (237) (2002) 1-25.

[52] O. Steinbach, B. I. Wohlmuth, L. Wunderlich, Trace and flux a priori error estimates in the finite element approximations of Signorini-type problems, IMA Journal of Numerical Analysis 36 (3) (2015) 1072-1095.

[53] J. L. Lions, E. Magenes, Problemes aux limites non homogenes et applications, Vol. 1, Dunod, 1968.

[54] H. Triebel, Interpolation theory, function spaces, differential operators, North-Holland, 1978. 